\title{
Anatomy of exotic Higgs decays in 2HDM
}

\author{
Felix Kling, ${ }^{a, b}$ Jose Miguel $\mathbf{N o}^{c}$ and Shufang $\mathbf{S u}^{a}$ \\ ${ }^{a}$ Department of Physics, University of Arizona, \\ 1118 E. 4th St., Tucson, Arizona 85721, U.S.A. \\ ${ }^{b}$ Fermilab, \\ P.O. Box 500, Batavia, IL 60510, U.S.A. \\ ${ }^{c}$ Department of Physics and Astronomy, University of Sussex, \\ Brington, BN1 9QH, U.K. \\ E-mail: kling@email.arizona.edu, J.M.No@sussex.ac.uk, \\ shufang@email.arizona.edu
}

\begin{abstract}
Large mass splittings between new scalars in two-Higgs-doublet models (2HDM) open a key avenue to search for these new states via exotic heavy Higgs decays. We discuss in detail the different search channels for these new scalars at the LHC in the presence of a sizable mass splitting, i.e. a hierarchical $2 \mathrm{HDM}$ scenario, taking into account the theoretical and experimental constraints. We provide benchmark planes to exploit the complementarity among these searches, analyzing their potential to probe the hierarchical 2HDM parameter space during LHC Run 2.
\end{abstract}

Keywords: Beyond Standard Model, Higgs Physics

ARXIV EPRINT: 1604.01406 


\section{Contents}

1 Introduction 1

2 Two Higgs doublet models: a review 2

2.1 2HDM Lagrangian and Higgs potential 2

2.2 Interactions in the $2 \mathrm{HDM} \quad 4$

2.3 The alignment limit and the role of $m_{12}^{2} \quad 5$

$3 \quad 2 H D M$ theoretical and experimental constraints $\quad 6$

$\begin{array}{lll}3.1 & \text { Vacuum stability } & 6\end{array}$

$\begin{array}{lll}3.2 & \text { Perturbativity and unitarity } & 6\end{array}$

$\begin{array}{lll}3.3 & \text { Electroweak precision measurements } & 8\end{array}$

3.4 Flavour constraints 8

3.5 LHC and LEP constraints 9

$\begin{array}{lll}3.6 & \text { From constraints to 2HDM benchmarks } & 10\end{array}$

4 LHC production and decay of 2HDM Higgses 11

5 2HDM planes for exotic Higgs decays $\quad 14$

$\begin{array}{ll}5.1 \text { Exotic decays in the alignment limit } & 16\end{array}$

5.1.1 BP IA: $m_{A}>m_{H}=m_{H^{ \pm}} \quad 16$

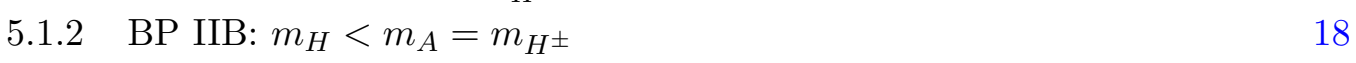

5.1.3 BP IB: $m_{A}<m_{H^{ \pm}}=m_{H} \quad 20$

5.1.4 BP IIA: $m_{H}>m_{A}=m_{H^{ \pm}} \quad 20$

$\begin{array}{lll}5.2 & \text { Exotic decays into } h \text { away from alignment } & 21\end{array}$

5.2.1 BP III: $m_{A}=m_{H}=m_{H^{ \pm}}$vs. $c_{\beta-\alpha} \quad 21$

6 Conclusions 23

A Production cross sections and branching ratios of 2HDM Higgses $\quad 26$

$\begin{array}{lll}\text { A.1 } 2 \mathrm{HDM} \text { production cross sections } & 26\end{array}$

A.2 2HDM branching ratios for exotic Higgs decays 27

\section{Introduction}

Analyses of the results from the LHC $7-8 \mathrm{TeV}$ run by both ATLAS and CMS show that the properties of the Higgs particle at $m_{h} \sim 125 \mathrm{GeV}$ are close to those expected for the Standard Model (SM) Higgs boson $h_{\mathrm{SM}}[1,2]$. The complete nature of the scalar sector responsible for electroweak (EW) symmetry-breaking, however, remains to be determined, and it is particularly interesting to ascertain whether the Higgs sector consists of only one 
$\mathrm{SU}(2)_{L}$ scalar doublet or has a richer structure containing additional states. Addressing this question is a key task for present and future studies at the Large Hadron Collider (LHC).

Two Higgs doublet models (2HDM) constitute the prime example of a well-motivated extended Higgs sector, appearing in many extensions of the SM such as the MSSM [3], composite Higgs models [4,5] and viable EW baryogenesis scenarios [6-9]. In addition to the SM-like CP-even Higgs boson, the 2HDM spectrum contains one more CP-even Higgs, a CP-odd Higgs and a pair of charged ones. ${ }^{1}$ In recent years, its allowed parameter space has been scrutinized in light of ATLAS/CMS Higgs coupling measurements and searches for extra Higgses at the LHC [10-19].

A key avenue to probe the 2HDM heavy Higgs bosons at the LHC which has started to attract attention recently is the search for exotic decays of the heavy Higgses in the presence of a sizable mass splitting among them [20-25] (see also [19]). These sizable splittings are difficult to realize in the MSSM, while in more general 2HDM scenarios they may lead to important physical consequences. ${ }^{2}$ While the conventional decay channels of a heavy Higgs into two SM quarks, leptons or gauge bosons have been the focus of most of the existing searches, the exotic (non-SM) modes of a heavy Higgs decaying into two light Higgses, or one light Higgs with one SM gauge boson quickly dominate once they are kinematically open. The current exclusion bounds on extra Higgses based on their conventional decays only will be therefore significantly relaxed. On the other hand, the exotic decay modes offer new discovery channels, which have already shown exclusion power during the $8 \mathrm{TeV}$ LHC run [26, 27], and yield very promising prospects for the $13 \mathrm{TeV}$ LHC run. In this work, we aim to provide a comprehensive categorization and analysis of the exotic search channels for the new 2HDM scalars, highlighting the complementarity among them, and provide guiding benchmark planes for Run 2 of the LHC at $13 \mathrm{TeV}$.

After a review of the $2 \mathrm{HDM}$ in section 2, we present the constraints on the 2HDM parameter space coming from theoretical considerations (stability of the EW minimum, perturbativity and tree-level unitarity) and experimental measurements in section 3 , where we also introduce the salient features of our benchmark scenarios for exotic 2HDM Higgs decays (section 3.6) motivated by the theoretical and experimental constraints. In section 4 we discuss the production and decay of non-SM Higgses at the LHC, and then analyze in depth our different benchmark scenarios in section 5 , before concluding in section 6 .

\section{Two Higgs doublet models: a review}

\subsection{HDM Lagrangian and Higgs potential}

In the $2 \mathrm{HDM}$, we introduce two $\mathrm{SU}(2)_{L}$ doublets $\Phi_{i}(i=1,2)$ :

$$
\Phi_{i}=\left(\begin{array}{c}
\phi_{i}^{+} \\
\left(v_{i}+\phi_{i}^{0}+i \varphi_{i}\right) / \sqrt{2}
\end{array}\right),
$$

\footnotetext{
${ }^{1}$ Here we take the assumption of a CP-conserving 2HDM. In the case of CP-violation, the three neutral Higgses are mixed together to form three mass eigenstates without definite CP properties.

${ }^{2}$ E.g. it has been shown in [22] that sizable mass splittings between the 2HDM new scalars favour a strong EW phase transition that could lead to baryogenesis.
} 
where $v_{i}$ are the vacuum expectation values (vev) of the neutral components, satisfying $v_{1}^{2}+v_{2}^{2}=v^{2}$, with $v=246 \mathrm{GeV}$. The ratio of vevs is defined as $\tan \beta \equiv v_{2} / v_{1}$. The $2 \mathrm{HDM}$ Lagrangian for $\Phi_{i}$ can be written as

$$
\mathcal{L}=\sum_{i}\left|D_{\mu} \Phi_{i}\right|^{2}-V\left(\Phi_{1}, \Phi_{2}\right)+\mathcal{L}_{\text {Yuk }}
$$

where the first term denotes the kinetic term for the two Higgs doublets, $V\left(\Phi_{1}, \Phi_{2}\right)$ is the Higgs potential and the last term denotes the Yukawa interactions between $\Phi_{i}$ and the SM fermions. Assuming CP conservation and a soft $\mathbb{Z}_{2}$ symmetry breaking, the 2 HDM Higgs potential can be written down as: ${ }^{3}$

$$
\begin{aligned}
V\left(\Phi_{1}, \Phi_{2}\right)= & m_{11}^{2} \Phi_{1}^{\dagger} \Phi_{1}+m_{22}^{2} \Phi_{2}^{\dagger} \Phi_{2}-m_{12}^{2}\left(\Phi_{1}^{\dagger} \Phi_{2}+\text { h.c. }\right)+\frac{\lambda_{1}}{2}\left(\Phi_{1}^{\dagger} \Phi_{1}\right)^{2}+\frac{\lambda_{2}}{2}\left(\Phi_{2}^{\dagger} \Phi_{2}\right)^{2} \\
& +\lambda_{3}\left(\Phi_{1}^{\dagger} \Phi_{1}\right)\left(\Phi_{2}^{\dagger} \Phi_{2}\right)+\lambda_{4}\left(\Phi_{1}^{\dagger} \Phi_{2}\right)\left(\Phi_{2}^{\dagger} \Phi_{1}\right)+\frac{1}{2}\left[\lambda_{5}\left(\Phi_{1}^{\dagger} \Phi_{2}\right)^{2}+\text { h.c. }\right]
\end{aligned}
$$

After EW symmetry breaking, the physical 2HDM scalar spectrum consists of five states: two CP-even Higgses $h, H$ with $m_{h}<m_{H}$, a CP-odd scalar $A$ and a charged scalar pair $H^{ \pm}[28]$, which may be written as

$$
\begin{aligned}
& \left(\begin{array}{c}
H \\
h
\end{array}\right)=\left(\begin{array}{cc}
c_{\alpha} & s_{\alpha} \\
-s_{\alpha} & c_{\alpha}
\end{array}\right)\left(\begin{array}{l}
\phi_{1}^{0} \\
\phi_{2}^{0}
\end{array}\right), \\
& \left(\begin{array}{c}
G \\
A
\end{array}\right)=\left(\begin{array}{cc}
c_{\beta} & s_{\beta} \\
-s_{\beta} & c_{\beta}
\end{array}\right)\left(\begin{array}{l}
\varphi_{1} \\
\varphi_{2}
\end{array}\right), \quad\left(\begin{array}{c}
G^{ \pm} \\
H^{ \pm}
\end{array}\right)=\left(\begin{array}{cc}
c_{\beta} & s_{\beta} \\
-s_{\beta} & c_{\beta}
\end{array}\right)\left(\begin{array}{c}
\phi_{1}^{ \pm} \\
\phi_{2}^{ \pm}
\end{array}\right),
\end{aligned}
$$

with the angle $\alpha$ parametrizing the mixing between the neutral CP-even components (we use the shorthand notation $\left.s_{x} \equiv \sin x, c_{x} \equiv \cos x, t_{x} \equiv \tan x\right)$. The Goldstone bosons $G$ and $G^{ \pm}$are absorbed as longitudinal components of the $Z$ and $W^{ \pm}$bosons. In the limit $c_{\beta-\alpha}=0$ (the alignment limit for $h$ ), the state $h$ can be identified with the SM Higgs, its couplings to fermions and gauge bosons being precisely those predicted by the SM. ${ }^{4}$ It is thus convenient to describe the model in terms of $t_{\beta}, c_{\beta-\alpha}$, the physical scalar masses $m_{h}, m_{H}, m_{A}, m_{H^{ \pm}}$, the soft $\mathbb{Z}_{2}$ symmetry breaking parameter $m_{12}^{2}$ and the vev $v$. The quartic couplings in eq. (2.3) can be expressed in terms of the physical masses and mixing angles as (see e.g. [30])

$$
\begin{aligned}
& v^{2} \lambda_{1}=\frac{m_{H}^{2} c_{\alpha}^{2}+m_{h}^{2} s_{\alpha}^{2}-m_{12}^{2} t_{\beta}}{c_{\beta}^{2}}, \quad v^{2} \lambda_{2}=\frac{m_{H}^{2} s_{\alpha}^{2}+m_{h}^{2} c_{\alpha}^{2}-m_{12}^{2} t_{\beta}^{-1}}{s_{\beta}^{2}}, \\
& v^{2} \lambda_{3}=\frac{\left(m_{H}^{2}-m_{h}^{2}\right) s_{\alpha} c_{\alpha}+2 m_{H^{ \pm}}^{2} s_{\beta} c_{\beta}-m_{12}^{2}}{s_{\beta} c_{\beta}}, \quad v^{2} \lambda_{4}=\frac{\left(m_{A}^{2}-2 m_{H^{ \pm}}^{2}\right) s_{\beta} c_{\beta}+m_{12}^{2}}{s_{\beta} c_{\beta}}, \\
& v^{2} \lambda_{5}=\frac{-m_{A}^{2} s_{\beta} c_{\beta}+m_{12}^{2}}{s_{\beta} c_{\beta}}
\end{aligned}
$$

\footnotetext{
${ }^{3}$ The most general scalar potential also contains the terms $\left[\lambda_{6}\left(\Phi_{1}^{\dagger} \Phi_{1}\right)+\lambda_{7}\left(\Phi_{2}^{\dagger} \Phi_{2}\right)\right]\left(\Phi_{1}^{\dagger} \Phi_{2}\right)+$ h.c. (leading to potentially dangerous flavour changing neutral currents), which can however be forbidden by imposing a $\mathbb{Z}_{2}$ symmetry, softly broken by the $m_{12}^{2}$ term.

${ }^{4}$ We note that if the heavier neutral CP even Higgs $H$ is identified with the observed $125 \mathrm{GeV}$ SM-like Higgs, the alignment limit is instead described by $s_{\beta-\alpha}=0$ [29].
} 


\subsection{Interactions in the $2 \mathrm{HDM}$}

The couplings of the CP-even scalars to a pair of gauge bosons, arising from the Higgs kinetic term in eq. (2.2), are [28]

$$
g_{h Z Z}=\frac{2 i m_{Z}^{2}}{v} s_{\beta-\alpha}, \quad g_{H Z Z}=\frac{2 i m_{Z}^{2}}{v} c_{\beta-\alpha}, \quad g_{h W W}=\frac{2 i m_{W}^{2}}{v} s_{\beta-\alpha}, \quad g_{H W W}=\frac{2 i m_{W}^{2}}{v} c_{\beta-\alpha} .
$$

The CP-odd scalar $A$ does not couple to pairs of vector bosons, while the charged scalar $H^{ \pm}$only couples to pair of vector bosons at loop level. In addition, the couplings of two scalars and one vector boson read

$$
\begin{array}{rlrl}
g_{h A Z} & =\frac{m_{Z}}{v} c_{\beta-\alpha}\left(p_{A}^{\mu}-p_{h}^{\mu}\right), & g_{H A Z} & =-\frac{m_{Z}}{v} s_{\beta-\alpha}\left(p_{A}^{\mu}-p_{H}^{\mu}\right), \\
g_{h H^{ \pm} W^{\mp}} & = \pm \frac{i m_{W}}{v} c_{\beta-\alpha}\left(p_{H^{+}}^{\mu}-p_{h}^{\mu}\right), & g_{H H^{ \pm} W^{\mp}}=\mp \frac{i m_{W}}{v} s_{\beta-\alpha}\left(p_{H^{+}}^{\mu}-p_{H}^{\mu}\right), \\
g_{A H^{ \pm} W^{\mp}} & =\frac{m_{W}}{v}\left(p_{H^{+}}^{\mu}-p_{A}^{\mu}\right),
\end{array}
$$

in which $p^{\mu}$ are the outgoing momentum for the corresponding particle. The $h H Z$-coupling is absent due to $\mathrm{CP}$ conservation. We note that, considering $h(H)$ to be the SM-like $125 \mathrm{GeV}$ Higgs with $c_{\beta-\alpha}=0\left(s_{\beta-\alpha}=0\right)$, gauge boson couplings to two non-SM like Higgses are unsuppressed, while the gauge boson couplings to $h(H)$ and one non-SM like Higgs are suppressed by $c_{\beta-\alpha}\left(s_{\beta-\alpha}\right)$.

Regarding the cubic couplings among scalars arising from the $2 \mathrm{HDM}$ scalar potential eq. (2.3), the relevant ones for our analysis are

$$
\begin{aligned}
g_{H h h} & =-\frac{1}{4 s_{2 \beta} v}\left(\frac{4 m_{12}^{2}}{s_{\beta} c_{\beta}}\left(c_{\beta-\alpha}^{2} s_{\beta+\alpha}-2 s_{\beta-\alpha} c_{\beta-\alpha} c_{\beta+\alpha}\right)-\left(2 m_{h}^{2}+m_{H}^{2}\right)\left(s_{3 \alpha-\beta}+s_{\alpha+\beta}\right)\right), \\
g_{H A A} & =-\frac{1}{4 s_{2 \beta} v}\left(\frac{4 m_{12}^{2}}{s_{\beta} c_{\beta}} s_{\beta+\alpha}-8 m_{A}^{2} c_{\beta-\alpha} s_{\beta} c_{\beta}-m_{H}^{2}\left(s_{\alpha-3 \beta}+3 s_{\alpha+\beta}\right)\right), \\
g_{H H^{+} H^{-}} & =-\frac{1}{4 s_{2 \beta} v}\left(\frac{4 m_{12}^{2}}{s_{\beta} c_{\beta}} s_{\beta+\alpha}-8 m_{H^{ \pm}}^{2} c_{\beta-\alpha} s_{\beta} c_{\beta}-m_{H}^{2}\left(s_{\alpha-3 \beta}+3 s_{\alpha+\beta}\right)\right),
\end{aligned}
$$

which could mediate decays with $H$ being the parent scalar: $H \rightarrow h h, H \rightarrow A A$ and $H \rightarrow H^{+} H^{-}$. As seen directly from eq. (2.8), these couplings depend not only on the mass spectrum, but also on the soft $\mathbb{Z}_{2}$ symmetry breaking term $m_{12}^{2}$ (we note here that the couplings shown in [28] assume the MSSM relation $m_{12}^{2}=m_{A}^{2} s_{\beta} c_{\beta}$ ). We also stress that for a light CP-odd scalar $A$ with $m_{A}<m_{h} / 2$, the decay channel $h \rightarrow A A$ could be open, being however very constrained experimentally ${ }^{5}$ (see [32] for a discussion of this region of the $2 \mathrm{HDM}$ parameter space).

Finally, as is well-known the couplings of the 2HDM scalars to SM fermions, contained in $\mathcal{L}_{\text {Yuk }}$ in eq. (2.2) are not univocally determined by the gauge structure of the model. In the presence of a $\mathbb{Z}_{2}$ symmetry guaranteeing the absence of tree-level flavour changing

\footnotetext{
${ }^{5}$ The possibility of a light charged scalar with $m_{H^{ \pm}}<m_{h} / 2$ has been ruled out experimentally by LEP, which puts a lower bound $m_{H^{ \pm}}>80 \mathrm{GeV}$ for Type II $\left(m_{H^{ \pm}}>72 \mathrm{GeV}\right.$ for Type I) 2HDM [31], thus forbidding the decay $h \rightarrow H^{+} H^{-}$.
} 


\begin{tabular}{|c|c|c|}
\hline State & Up-type fermions & Down-type fermions \\
\hline$h$ & $c_{\alpha} / s_{\beta}=s_{\beta-\alpha}+c_{\beta-\alpha} / t_{\beta}$ & $-s_{\alpha} / c_{\beta}=s_{\beta-\alpha}-c_{\beta-\alpha} t_{\beta}$ \\
\hline$H$ & $s_{\alpha} / s_{\beta}=c_{\beta-\alpha}-s_{\beta-\alpha} / t_{\beta}$ & $c_{\alpha} / c_{\beta}=c_{\beta-\alpha}+s_{\beta-\alpha} t_{\beta}$ \\
\hline$A$ & $1 / t_{\beta}$ & $t_{\beta}$ \\
\hline
\end{tabular}

Table 1. Tree-level couplings to up-type fermions and down-type fermions normalized to their SM values for $h, H$ and $A$ in the Type II 2HDM.

neutral currents [33], four possible 2HDM types exist (see [34] for a discussion). The couplings of the neutral scalar states to SM fermions, normalized to their SM values, can be expressed in terms of functions of $\alpha$ and $\beta$, shown in table 1 for the particular case of a Type II $2 \mathrm{HDM}$ (one Higgs doublet $\Phi_{2}$ couples to the up-type quarks, while the other Higgs doublet $\Phi_{1}$ couples to the down-type quarks and leptons).

\subsection{The alignment limit and the role of $m_{12}^{2}$}

It is useful to cast the relations between the quartic couplings and the physical masses eq. (2.5) in terms of $c_{\beta-\alpha}$, which characterizes the departure from the alignment limit for $h$

$$
\begin{aligned}
& v^{2} \lambda_{1}=m_{h}^{2}-\frac{t_{\beta}\left(m_{12}^{2}-m_{H}^{2} s_{\beta} c_{\beta}\right)}{c_{\beta}^{2}}+\left(m_{h}^{2}-m_{H}^{2}\right)\left[c_{\beta-\alpha}^{2}\left(t_{\beta}^{2}-1\right)-2 t_{\beta} s_{\beta-\alpha} c_{\beta-\alpha}\right], \\
& v^{2} \lambda_{2}=m_{h}^{2}-\frac{\left(m_{12}^{2}-m_{H}^{2} s_{\beta} c_{\beta}\right)}{t_{\beta} s_{\beta}^{2}}+\left(m_{h}^{2}-m_{H}^{2}\right)\left[c_{\beta-\alpha}^{2}\left(t_{\beta}^{-2}-1\right)+2 t_{\beta}^{-1} s_{\beta-\alpha} c_{\beta-\alpha}\right], \\
& v^{2} \lambda_{3}=m_{h}^{2}+2 m_{H^{ \pm}}^{2}-2 m_{H}^{2}-\frac{\left(m_{12}^{2}-m_{H}^{2} s_{\beta} c_{\beta}\right)}{s_{\beta} c_{\beta}}-\left(m_{h}^{2}-m_{H}^{2}\right)\left[2 c_{\beta-\alpha}^{2}+s_{\beta-\alpha} c_{\beta-\alpha}\left(t_{\beta}-t_{\beta}^{-1}\right)\right], \\
& v^{2} \lambda_{4}=m_{A}^{2}-2 m_{H^{ \pm}}^{2}+m_{H}^{2}+\frac{\left(m_{12}^{2}-m_{H}^{2} s_{\beta} c_{\beta}\right)}{s_{\beta} c_{\beta}} \\
& v^{2} \lambda_{5}=m_{H}^{2}-m_{A}^{2}+\frac{\left(m_{12}^{2}-m_{H}^{2} s_{\beta} c_{\beta}\right)}{s_{\beta} c_{\beta}} .
\end{aligned}
$$

Current data from LHC Run 1 favour the alignment limit $c_{\beta-\alpha}=0$ [35] (see also [1012, 14-16, 18]). For a Type II $2 \mathrm{HDM}$ the only other allowed possibility is the wrong-sign scenario [36] $s_{\beta+\alpha} \simeq 1$ (compatible with measurements of Higgs signal strengths for $t_{\beta}>3$ ). For $c_{\beta-\alpha}=0$, the relations eq. (2.9) simply become

$$
\begin{aligned}
& v^{2} \lambda_{1}=m_{h}^{2}-\frac{t_{\beta}\left(m_{12}^{2}-m_{H}^{2} s_{\beta} c_{\beta}\right)}{c_{\beta}^{2}}, \\
& v^{2} \lambda_{2}=m_{h}^{2}-\frac{\left(m_{12}^{2}-m_{H}^{2} s_{\beta} c_{\beta}\right)}{t_{\beta} s_{\beta}^{2}} \\
& v^{2} \lambda_{3}=m_{h}^{2}+2 m_{H^{ \pm}}^{2}-2 m_{H}^{2}-\frac{\left(m_{12}^{2}-m_{H}^{2} s_{\beta} c_{\beta}\right)}{s_{\beta} c_{\beta}}, \\
& v^{2} \lambda_{4}=m_{A}^{2}-2 m_{H^{ \pm}}^{2}+m_{H}^{2}+\frac{\left(m_{12}^{2}-m_{H}^{2} s_{\beta} c_{\beta}\right)}{s_{\beta} c_{\beta}} \\
& v^{2} \lambda_{5}=m_{H}^{2}-m_{A}^{2}+\frac{\left(m_{12}^{2}-m_{H}^{2} s_{\beta} c_{\beta}\right)}{s_{\beta} c_{\beta}}
\end{aligned}
$$


The combination $m_{12}^{2}-m_{H}^{2} s_{\beta} c_{\beta}$ in eq. (2.10) will play a key role in the following discussion: the value of $m_{12}^{2}$ is not fixed by the mass spectrum or the scalar couplings to gauge bosons and fermions, only entering the trilinear scalar couplings eq. (2.8). Its possible allowed values are dictated by theoretical constraints on the $2 \mathrm{HDM}$ parameter space, namely the boundedness from below of the scalar potential eq. (2.3) and the stability of the EW minimum, and the requirements of perturbativity and tree-level unitarity on the quartic couplings $\lambda_{i}$, as shown in the next section. These have a large impact on the allowed values of masses $m_{H}, m_{A}, m_{H^{ \pm}}, m_{12}^{2}$ and $t_{\beta}$ (and $c_{\beta-\alpha}$ away from alignment), as the absence of a value of $m_{12}^{2}$ satisfying the theoretical constraints for a given set of values for $m_{H}, m_{A}$, $m_{H^{ \pm}}$and $t_{\beta}$, indicates that such set of values is not physically viable (see e.g. [19]).

\section{$3 \quad 2 H D M$ theoretical and experimental constraints}

\subsection{Vacuum stability}

In order to have a stable vacuum, the following conditions need to be fulfilled [30]

$$
\lambda_{1}>0, \quad \lambda_{2}>0, \quad \lambda_{3}>-\sqrt{\lambda_{1} \lambda_{2}}, \quad \lambda_{3}+\lambda_{4}-\left|\lambda_{5}\right|>-\sqrt{\lambda_{1} \lambda_{2}} .
$$

For $c_{\beta-\alpha}=0$, satisfying the first two conditions requires $m_{12}^{2}-m_{H}^{2} s_{\beta} c_{\beta} \lesssim 0$ for either $t_{\beta}>1$ or $t_{\beta}<1$, as seen from eq. (2.10). Moreover, eq. (2.9) shows that a departure from alignment generically has a negative impact on the first two stability conditions. Focusing on the alignment limit, the first two requirements are automatically satisfied for $m_{12}^{2}-m_{H}^{2} s_{\beta} c_{\beta}=0$, with the last two given by

$$
m_{h}^{2}+m_{H^{ \pm}}^{2}-m_{H}^{2}>0 \quad, \quad m_{h}^{2}+m_{A}^{2}-m_{H}^{2}>0 .
$$

This implies that for $m_{H}>m_{A}, m_{H^{ \pm}}$, the mass splittings between the heavy CP-even Higgs $H$ and the other heavy scalars $A$ and $H^{ \pm}$have to be small, such that the decays of $H$ into $A Z, A A, H^{+} H^{-}$or $H^{ \pm} W^{\mp}$ are not kinematically allowed. For $m_{12}^{2}=0$ all four stability conditions of eq. (3.1) are automatically fulfilled. The allowed region in the $m_{12}$ vs. $t_{\beta}$ plane is shown in the left panel of figure 1 for $m_{A}=m_{H^{ \pm}}=400 \mathrm{GeV}$ and $m_{H}=200,300,400 \mathrm{GeV}$ as an illustration. As seen from figure 1 , the regions $m_{12}^{2}<m_{H}^{2} s_{\beta} c_{\beta}$ are generically allowed by the vacuum stability requirement.

\subsection{Perturbativity and unitarity}

Upon imposing the perturbativity condition $\left|\lambda_{i}\right| \leq 4 \pi$, the strongest constraints in the alignment limit come respectively from $v^{2} \lambda_{1} \sim t_{\beta}^{3}\left(m_{12}^{2}-m_{H}^{2} s_{\beta} c_{\beta}\right)$ for $t_{\beta} \gg 1$ and $v^{2} \lambda_{2} \sim$ $t_{\beta}^{-3}\left(m_{12}^{2}-m_{H}^{2} s_{\beta} c_{\beta}\right)$ for $t_{\beta} \ll 1$. Thus, perturbativity requires $\left|m_{12}^{2}-m_{H}^{2} s_{\beta} c_{\beta}\right| \lesssim v^{2}$ unless $t_{\beta} \sim 1$. Moreover, even for $m_{12}^{2}=m_{H}^{2} s_{\beta} c_{\beta}$, perturbativity of $\lambda_{3-5}$ imposes constraints on the size of the mass splittings among the new scalars. 

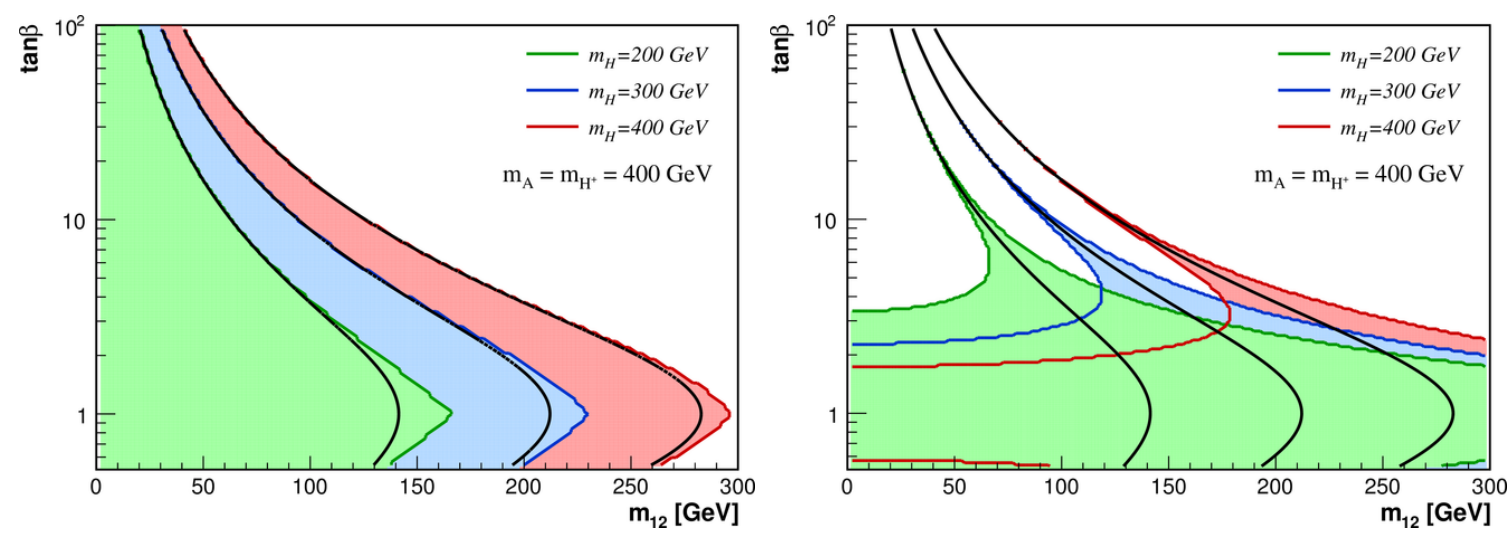

Figure 1. Allowed region in the $\left(m_{12}, t_{\beta}\right)$ plane from vacuum stability (left panel) and unitarity $\left|\Lambda_{i}\right|<8 \pi$ (right panel) for $m_{H}=400 \mathrm{GeV}$ (red), $300 \mathrm{GeV}$ (blue) and $200 \mathrm{GeV}$ (green), assuming $c_{\beta-\alpha}=0$ and $m_{A}=m_{H^{ \pm}}=400 \mathrm{GeV}$. The black lines denote the relation $m_{12}^{2}=m_{H}^{2} s_{\beta} c_{\beta}$.

Even stronger constraints are found when requiring tree-level unitarity ${ }^{6}$ of the scattering matrix in the 2HDM scalar sector [37]. The eigenvalues of the scattering matrix read

$$
\begin{aligned}
\Lambda_{1,2} & =\lambda_{3} \pm \lambda_{4} \\
\Lambda_{3,4} & =\lambda_{3} \pm \lambda_{5} \\
\Lambda_{5,6} & =\lambda_{3}+2 \lambda_{4} \pm 3 \lambda_{5} \\
\Lambda_{7,8} & =\frac{1}{2}\left(\lambda_{1}+\lambda_{2} \pm \sqrt{\left(\lambda_{1}-\lambda_{2}\right)^{2}+4 \lambda_{4}^{2}}\right) \\
\Lambda_{9,10} & =\frac{1}{2}\left(\lambda_{1}+\lambda_{2} \pm \sqrt{\left(\lambda_{1}-\lambda_{2}\right)^{2}+4\left|\lambda_{5}\right|^{2}}\right), \\
\Lambda_{11,12} & =\frac{1}{2}\left(3\left(\lambda_{1}+\lambda_{2}\right) \pm \sqrt{9\left(\lambda_{1}-\lambda_{2}\right)^{2}+4\left(2 \lambda_{3}+\lambda_{4}\right)^{2}}\right) .
\end{aligned}
$$

Performing a partial-wave expansion of the scattering amplitudes yields limits on the partial wave amplitudes, which for the $J=0$ case translate into the constraint $\left|\operatorname{Re}\left(\Lambda_{i}\right)\right|<8 \pi$ (see e.g. [38]), which we consider here. A quick inspection of eq. (3.3) shows that for $t_{\beta} \gg 1$ the scattering matrix eigenvalues scale as $\Lambda_{7,9,11} \sim \lambda_{1}$ (particularly $\Lambda_{11} \simeq 3 \lambda_{1}$ ), which again imposes $\left|m_{12}^{2}-m_{H}^{2} s_{\beta} c_{\beta}\right| \lesssim v^{2}$ (and yields an even stronger constraint than the perturbativity one). A similar argument follows for $t_{\beta} \ll 1$, this time with $\Lambda_{7,9,11} \sim \lambda_{2}$. As a result, $m_{12}^{2} \approx m_{H}^{2} s_{\beta} c_{\beta}$ is strongly preferred unless $t_{\beta} \sim 1$, as shown explicitly in the right panel of figure 1 (for $m_{A}=m_{H^{ \pm}}$). In the limit $m_{12}^{2}=m_{H}^{2} s_{\beta} c_{\beta}$, the scattering matrix eigenvalues from eq. (3.3) become independent of $t_{\beta}$ (in alignment $c_{\beta-\alpha}=0$ ) and read

$$
\begin{aligned}
\Lambda_{1(9), 10} v^{2} & =m_{h}^{2} \mp m_{H}^{2} \pm m_{A}^{2}, & \Lambda_{2} v^{2} & =m_{h}^{2}-3 m_{H}^{2}-m_{A}^{2}+4 m_{H^{ \pm}}^{2}, \\
\Lambda_{3} v^{2} & =m_{h}^{2}-m_{H}^{2}-m_{A}^{2}+2 m_{H^{ \pm}}^{2}, & \Lambda_{4,5} v^{2} & =m_{h}^{2} \mp 3 m_{H}^{2} \pm m_{A}^{2} \pm 2 m_{H^{ \pm}}^{2}, \\
\Lambda_{6} v^{2} & =m_{h}^{2}-3 m_{H}^{2}+5 m_{A}^{2}-2 m_{H^{ \pm}}^{2}, & \Lambda_{7,8} v^{2} & =m_{h}^{2} \pm m_{H}^{2} \pm m_{A}^{2} \mp 2 m_{H^{ \pm}}^{2}, \\
\Lambda_{11} v^{2} & =5 m_{h}^{2}-3 m_{H}^{2}+m_{A}^{2}+2 m_{H^{ \pm}}^{2}, & \Lambda_{12} v^{2} & =m_{h}^{2}+3 m_{H}^{2}-m_{A}^{2}-2 m_{H^{ \pm}}^{2},
\end{aligned}
$$

\footnotetext{
${ }^{6}$ An analysis of unitarity constraints at one-loop level has been performed in [38].
} 

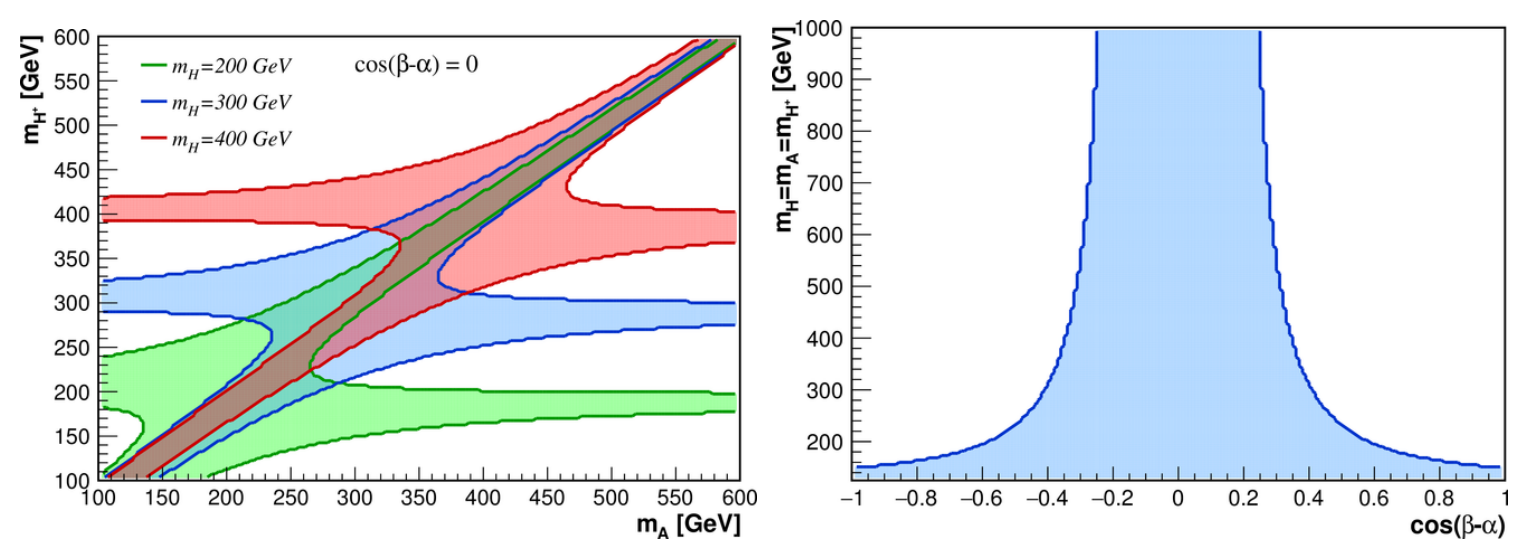

Figure 2. Left: $2 \mathrm{HDM}$ parameter space in the $\left(m_{A}, m_{H^{ \pm}}\right)$plane allowed at $95 \%$ C.L. by $S$ and $T$ measurements [39], for $m_{H}=400 \mathrm{GeV}$ (red), $m_{H}=300 \mathrm{GeV}$ (blue) and $m_{H}=200 \mathrm{GeV}$ (green), assuming $c_{\beta-\alpha}=0$. Right: $S-T$ constraints in the $\left(c_{\beta-\alpha}, m_{H}\right)$ plane for $m_{H}=m_{A}=m_{H^{ \pm}}$.

such that $\left|\Lambda_{i}\right|<8 \pi$ (note that $\Lambda_{i}$ are real) impose upper limits on the mass splittings (although not on the masses themselves). We also note that for $m_{12}^{2}=0, \Lambda_{1-6}$ are independent of $t_{\beta}$ (depending only on the scalar masses) while $\Lambda_{7-12}$ do depend on $t_{\beta}$, which once again results in $t_{\beta} \approx 1$ being the only accessible region for large mass splittings in this case.

\subsection{Electroweak precision measurements}

Measurements of EW precision observables (EWPO) impose strong constraints on the 2HDM mass spectrum. Adopting the current 95\% C.L. constraints on the $S$ and $T$ oblique parameters (with $U=0$ ) [39], the allowed region of parameter space in the $\left(m_{A}, m_{H^{ \pm}}\right.$) plane is shown, for $c_{\beta-\alpha}=0$ (neither $t_{\beta}$ nor $m_{12}^{2}$ affect $S$ and $T$ ), in the left panel of figure 2 respectively for $m_{H}=400 \mathrm{GeV}$ (red), $m_{H}=300 \mathrm{GeV}$ (blue) and $m_{H}=200 \mathrm{GeV}$ (green). Satisfying EWPO constraints requires the charged scalar mass to be close to one of the heavy neutral scalar masses: $m_{H^{ \pm}} \approx m_{H}$ or $m_{H^{ \pm}} \approx m_{A}$.

Away from the alignment limit, additional contributions to $S$ and $T$ proportional to $c_{\beta-\alpha}$ appear [28] (see also [40]), such that the scenario $m_{H}=m_{A}=m_{H^{ \pm}}$is only allowed for small $\left|c_{\beta-\alpha}\right|$ once $m_{H} \gg v$ is realized, as shown in the right panel of figure 2. The departure from alignment also allows for mild mass splittings among all the new scalars (e.g. $m_{A}>m_{H}+m_{Z}$ and $m_{H} \gtrsim m_{H^{ \pm}}+m_{W}$ ), which however does not significantly alter the phenomenology of exotic Higgs decays at the LHC, discussed in detail in section 5.

\subsection{Flavour constraints}

Various flavour measurements [41] provide indirect constraints on the charged scalar mass $m_{H^{ \pm}}$as a function of $t_{\beta}$. The different limits are computed for the case of a Type II 2HDM with SuperIso [42,43], and shown in the left panel of figure 3. The most stringent of these $^{7}$ comes from the measurement of the branching fraction (BR) of $b \rightarrow s \gamma\left(B_{d}^{0} \rightarrow X_{s} \gamma\right)$,

\footnotetext{
${ }^{7}$ We note here that the recent measurement from the BaBar Collaboration of the ratios of $B \rightarrow D^{*} \tau \nu$ to $B \rightarrow D^{*} \ell \nu$ decays and $B \rightarrow D \tau \nu$ to $B \rightarrow D \ell \nu$ decays cannot be accommodated within the Type II $2 \mathrm{HDM}$ [44]. However, a new measurement of the former ratio by the Belle Collaboration [45, 46] is in tension with this conclusion. Since this matter is not settled yet, we choose not to include these flavour measurements in our discussion.
} 

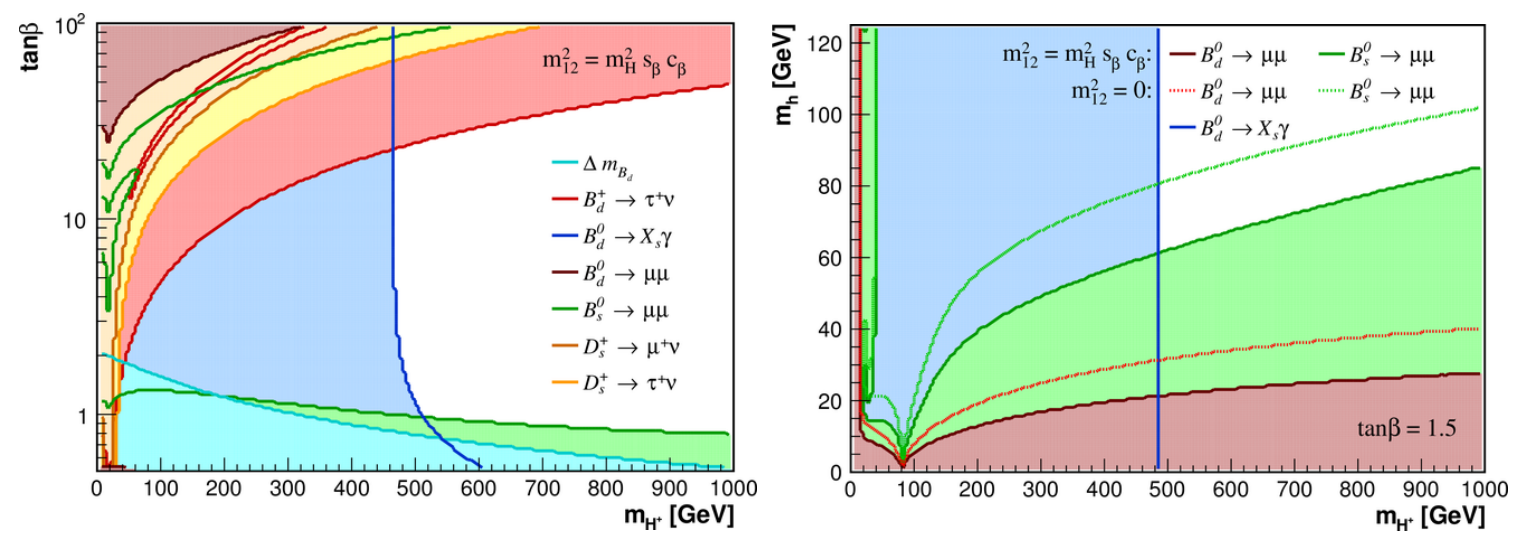

Figure 3. Type II 2HDM parameter space excluded by flavour constraints (see text for details).

which sets a limit $m_{H^{ \pm}}>480 \mathrm{GeV}$ at $95 \%$ C.L. [47] (we note that the limit is even stronger for $\left.t_{\beta}<2\right)$. For large $t_{\beta} \gtrsim 20$, the lower limit on $m_{H^{ \pm}}$set by the measurement of the branching fraction $B_{d}^{+} \rightarrow \tau^{+} \nu$ is significantly stronger, with $m_{H^{ \pm}} \gtrsim 700 \mathrm{GeV}$ for $t_{\beta}=30$. Similarly, the region $t_{\beta} \lesssim 1$ is very strongly constrained by $B_{s}^{0} \rightarrow \mu \mu$ and $\Delta m_{B_{d}}$.

For $m_{H}=125 \mathrm{GeV}$ and $s_{\beta-\alpha}=0$, when the heavy CP-even scalar $H$ is the SM-like Higgs, the mass of the light CP-even Higgs $h$ is constrained by flavour measurements as well, as shown in the right panel of figure 3 for Type II 2HDM. The strongest constraint in this case comes from $B_{s}^{0} \rightarrow \mu^{+} \mu^{-}$, which can exclude up to $m_{h}<100 \mathrm{GeV}$ (the precise bound depending on $m_{12}^{2}$ and $t_{\beta}$ ) for masses $m_{H^{ \pm}}$satisfying the $b \rightarrow s \gamma$ constraint.

Note that flavour constraints are typically very model dependent. Contributions from additional sectors in the model could relax the constraints, as has e.g. been studied in the MSSM framework for $b \rightarrow s \gamma$ [48]. Being mostly focused on the collider aspects of 2HDM Higgses, we will not consider flavour as a hard constraint in the following, however indicating its effect on the parameter space under consideration.

\subsection{LHC and LEP constraints}

We now review the constraints from direct searches of the new scalars. Besides the LEP bound $m_{H^{ \pm}}>80 \mathrm{GeV}(72 \mathrm{GeV})$ for Type II (I) $2 \mathrm{HDM}$ [31], LEP searches for $e^{+} e^{-} \rightarrow$ $A H(H \rightarrow b b / \tau \tau, A \rightarrow b b / \tau \tau)$ constrain the sum of the masses $m_{A}+m_{H} \gtrsim 209 \mathrm{GeV}$ [49]. At the LHC, the searches for $A / H$ in $b b$-associated production and decaying to $\tau \tau$ by ATLAS/CMS $[50,51]$ constrain the high $t_{\beta}$ region in the Type II 2HDM. Away from alignment, searches by ATLAS/CMS for $H \rightarrow W^{+} W^{-}, Z Z[52-54], A \rightarrow h Z(h \rightarrow b b)[55,56]$ and $H \rightarrow h h \rightarrow b b \gamma \gamma, b b b b$ [57-59] yield strong constraints on the $\left(c_{\beta-\alpha}, t_{\beta}\right)$ plane as a function of the respective mass $m_{H} / m_{A}$ (see e.g. [17, 19]). We however stress that the limits summarized above can be significantly weakened once exotic Higgs decay channels are open [19-21, 23, 25]). Searches for these new channels, e.g. via $A / H \rightarrow H Z / A Z$ are then crucial for probing $2 \mathrm{HDM}$ scenarios with large mass splittings among the new states (i.e. hierarchical $2 \mathrm{HDM}$ scenarios), and there is already ongoing effort by CMS in this direction [26, 27]. 
Finally, ATLAS/CMS searches impose constraints on the charged scalar [60, 61] beyond those of LEP. A light charged scalar $m_{H^{ \pm}} \lesssim m_{t}$ is mostly excluded by the non-observation of the decay $t \rightarrow H^{+} b \rightarrow \tau \nu b$ where the top is produced in top pair production. For $m_{H^{ \pm}}>m_{t}$, the current limit is very weak and only constrains the high $t_{\beta}$ region for $m_{H^{ \pm}}$ not much above the top mass (see [23] for a detailed discussion).

\subsection{From constraints to 2HDM benchmarks}

The combination of previous constraints provides a key guideline to the design of simplified $2 \mathrm{HDM}$ benchmark scenarios for LHC Run 2 searches at $13 \mathrm{TeV}$. EWPO measurements require the mass of the charged scalar to be close to the mass of one of the neutral scalars, and so we fix $m_{H^{ \pm}}=m_{H}$ or $m_{H^{ \pm}}=m_{A}$ in the following. In addition, measurements of Higgs signal strengths at the LHC favour the alignment limit $\left(c_{\beta-\alpha}=0\right.$ if $h$ is the $125 \mathrm{GeV}$ SM-like Higgs), particularly for Type II 2HDM. We then focus our analysis mostly on the alignment limit, and only consider deviations from alignment when discussing possible decays of the new scalars into the SM-like Higgs $h$.

Regarding the impact of theoretical constraints on the 2HDM parameter space, the previous discussion shows that satisfying unitarity/perturbativity and vacuum stability bounds (close to the alignment limit) for arbitrary values of $t_{\beta}$ requires $m_{12}^{2}=m_{H}^{2} s_{\beta} c_{\beta}$ and $m_{H} \lesssim m_{A}, m_{H^{ \pm}}$. Alternatively, stability is satisfied for any mass ordering if $m_{12}^{2}=0$, while unitarity requires in this case a low value of $t_{\beta}$. We thus consider these two scenarios as benchmark cases for our analysis:

- Case 1: $m_{12}^{2}=m_{H}^{2} s_{\beta} c_{\beta}{ }^{8}$

- From eq. (3.2), vacuum stability requires $m_{H} \lesssim m_{A}$ and $m_{H} \lesssim m_{H^{+}}$, and thus the exotic decays $H \rightarrow A Z$ and $H \rightarrow H^{ \pm} W^{\mp}$ are not kinematically allowed.

- Unitarity requires $\left|\Lambda_{i}\right|<8 \pi$, constraining the mass differences among the new scalar states (but not the absolute mass values). In particular, using eq. (3.4) we obtain the bound $\left|5\left(m_{A}^{2}-m_{H}^{2}\right)+m_{h}^{2}\right|<8 \pi v^{2}$ if $m_{H^{ \pm}}=m_{H}$, and the bound $\left|3\left(m_{A}^{2}-m_{H}^{2}\right)+5 m_{h}^{2}\right|<8 \pi v^{2}$ if $m_{H^{ \pm}}=m_{A}$.

- The cubic scalar couplings eq. (2.8) now read

$$
\begin{aligned}
g_{H h h} & =-\frac{c_{\beta-\alpha}}{s_{2 \beta} v}\left[2\left(m_{H}^{2}+m_{h}^{2}\right) s_{2 \alpha}-m_{H}^{2} s_{2 \beta}\right] \\
g_{H A A} & =-\frac{c_{\beta-\alpha}}{2 v}\left(m_{H}^{2}-2 m_{A}^{2}\right) \\
g_{H H^{+} H^{-}} & =-\frac{c_{\beta-\alpha}}{2 v}\left(m_{H}^{2}-2 m_{H^{ \pm}}^{2}\right) .
\end{aligned}
$$

In the alignment limit $c_{\beta-\alpha}=0$ all these couplings vanish, and therefore the decays $H \rightarrow A A, H \rightarrow H^{+} H^{-}$and $H \rightarrow h h$ are absent $(H \rightarrow A A$ and $H \rightarrow H^{+} H^{-}$are also not kinematically allowed for $\left.m_{12}^{2}=m_{H}^{2} s_{\beta} c_{\beta}\right)$.

\footnotetext{
${ }^{8}$ The authors of [62] have shown that this case is preferred when requiring the 2 HDM to be stable up to the Planck scale.
} 
- Case 2: $m_{12}^{2}=0$ and $t_{\beta} \sim 1 .^{9}$

- Vacuum stability does not constrain the parameter space. In particular $m_{H}>$ $m_{A}, m_{H^{+}}$is now possible, allowing the decays $H \rightarrow A Z$ and $H \rightarrow H^{ \pm} W^{\mp}$ (and potentially also $H \rightarrow A A$ and $H \rightarrow H^{+} H^{-}$).

- Unitarity imposes an upper bound on the scalar masses (not only on the mass splittings). This bound scales as $t_{\beta}^{-2}$ for $t_{\beta}>1$ and as $t_{\beta}^{2}$ for $t_{\beta}<1$, such that only the region $t_{\beta} \sim 1$ is allowed (we recall that in Type II $2 \mathrm{HDM}$, at least one of the neutral scalars needs to be heavy due to the combination of EWPO and flavour constraints).

- The cubic scalar couplings eq. (2.8) now read

$$
\begin{aligned}
g_{H h h} & =\frac{c_{\beta-\alpha}}{2 s_{2 \beta} v}\left(2 m_{h}^{2}+m_{H}^{2}\right)\left[\left(c_{\beta-\alpha}^{2}-s_{\beta-\alpha}^{2}\right) s_{2 \beta}-2 s_{\beta-\alpha} c_{\beta-\alpha} c_{2 \beta}\right] \\
g_{H A A} & =-\frac{1}{2 s_{2 \beta} v}\left[\left(m_{H}^{2}-2 m_{A}^{2}\right) c_{\beta-\alpha} s_{2 \beta}+2 m_{H}^{2} s_{\beta-\alpha} c_{2 \beta}\right] \\
g_{H H^{+} H^{-}} & =-\frac{1}{2 s_{2 \beta} v}\left[\left(m_{H}^{2}-2 m_{H^{ \pm}}^{2}\right) c_{\beta-\alpha} s_{2 \beta}+2 m_{H}^{2} s_{\beta-\alpha} c_{2 \beta}\right] .
\end{aligned}
$$

In the alignment limit the coupling $g_{H h h}$ vanishes and thus the decay $H \rightarrow h h$ is absent. However, the couplings $g_{H A A}$ and $g_{H H^{+} H^{-}}$are non-vanishing as long as $t_{\beta} \neq 1$.

For our analysis of benchmark scenarios away from alignment, which focus on the decays of $A, H, H^{ \pm}$into the SM-like Higgs $h$, we consider the same two cases above for consistency (even though these cases are motivated by theoretical constraints for $c_{\beta-\alpha}=0$ ).

\section{LHC production and decay of 2HDM Higgses}

We now discuss the salient features of the production and decay of the new 2HDM scalars at the LHC. The production of the CP-even and CP-odd neutral scalars $H, A$ at the $13 \mathrm{TeV}$ LHC occurs via gluon fusion $(g g \rightarrow H / A)$ and $b b$-associated production. Gluon fusion is the dominant production mechanism for small and moderate values of $t_{\beta}$, while for Type II $2 \mathrm{HDM}, b b$-associated production dominates at large $t_{\beta}$. In both cases, we compute the production cross section for $H$ and $A$ at NNLO in QCD via SusHi [64] (for $H$, the cross section does depend on $c_{\beta-\alpha}$, and in that case we consider the alignment limit $\left.c_{\beta-\alpha}=0\right)$. For the charged scalar $H^{ \pm}$, the dominant production mode for $m_{H^{ \pm}}>m_{t}$ is in association with a $t b$ pair, and we use the NLO cross section values provided by the Higgs Cross section Working Group (HXSWG) for $m_{H^{ \pm}}>200 \mathrm{GeV}$ [65-68]. A light charged scalar $\left(m_{H^{ \pm}}<m_{t}\right)$ is mainly produced through top quark decays $t \rightarrow H^{+} b$, and we use TOP ++2.0 [69] to compute the top pair production to NNLO in QCD, assuming a topquark mass $m_{t}=172.5 \mathrm{GeV}$. The LHC production cross sections for $H, A$ and $H^{+}$at $13 \mathrm{TeV}$ are shown in appendix A.

\footnotetext{
${ }^{9}$ This case has also been analyzed in [63].
} 


\begin{tabular}{|c|c|l|l|}
\hline Parent Scalar & Decay & \multicolumn{1}{|c|}{ Possible Final States } & \multicolumn{1}{|c|}{ Channels in 2HDM } \\
\hline \multirow{4}{*}{$\begin{array}{c}\text { Neutral } \\
H, A\end{array}$} & $H_{i} H_{i}$ & $(b b / \tau \tau / W W / Z Z / \gamma \gamma)(b b / \tau \tau / W W / Z Z / \gamma \gamma)$ & $H \rightarrow A A, h h$ \\
\cline { 2 - 4 } & $H_{i} Z$ & $(b b / \tau \tau / W W / Z Z / \gamma \gamma)(\ell \ell / q q / \nu \nu)$ & $H \rightarrow A Z, A \rightarrow H Z, h Z$ \\
\cline { 2 - 4 } & $H^{+} H^{-}$ & $(t b / \tau \nu / c s)(t b / \tau \nu / c s)$ & $H \rightarrow H^{+} H^{-}$ \\
\cline { 2 - 4 } & $H^{ \pm} W^{\mp}$ & $(t b / \tau \nu / c s)\left(\ell \nu / q q^{\prime}\right)$ & $H / A \rightarrow H^{ \pm} W^{\mp}$ \\
\hline Charged $H^{ \pm}$ & $H_{i} W^{ \pm}$ & $(b b / \tau \tau / W W / Z Z / \gamma \gamma)\left(\ell \nu / q q^{\prime}\right)$ & $H^{ \pm} \rightarrow h W^{ \pm}, H W^{ \pm}, A W^{ \pm}$ \\
\hline
\end{tabular}

Table 2. Summary of exotic decay modes for non-SM Higgs bosons. For each type of exotic decays (second column), we present possible final states (third column) and relevant channels in 2HDM (fourth column). In the second column, $H_{i}=h, H, A$.

Regarding the decays of the new scalars, in the alignment limit $c_{\beta-\alpha}=0$ the conventional (SM-like) decays of $A$ and $H$ are into $t t$ (if kinematically accessible), $b b, c c, \tau \tau$, and with a highly suppressed branching fraction into $g g, \gamma \gamma$ and $\mu \mu$. When open, the decay into $t t$ is dominant for low and moderate $t_{\beta}$, followed by the decay into $b b$. At high $t_{\beta}$, for Type II $2 \mathrm{HDM}$, the decay into $\tau \tau$ becomes important, where the decay into $b b$ can dominate even above the $t t$ threshold. For the CP-even Higgs $H$, the decay into massive gauge bosons $W^{+} W^{-}$and $Z Z$ is present away from the alignment limit, and dominates as soon as the departure from alignment is sizeable. For the charged scalar, the decay $H^{ \pm} \rightarrow t b$ dominates once it is kinematically open, followed by $H^{ \pm} \rightarrow \tau \nu, c s$ and $c b$. In the following, we compute all $2 \mathrm{HDM}$ branching fractions using $2 \mathrm{HDMC}[70]$.

We here stress that the above conventional decays of the new 2HDM scalars become suppressed once exotic (non SM-like) decay modes open up. These can be decays involving several states among $H, A, H^{ \pm}$, in the presence of a large mass splitting among the new scalars (see e.g. [20-25] for existing studies on individual channels), and/or decays into the SM-like Higgs boson $h$, namely $H \rightarrow h h, A \rightarrow h Z, H^{ \pm} \rightarrow h W^{ \pm}$, which are possible for $c_{\beta-\alpha} \neq 0$ and are also considered in the following as exotic (despite involving SM decay products) as they don't occur in the SM. In the former case, we can further distinguish between the decay of a new scalar into another one and a gauge boson, and the potential decays of $H$ into either $A A$ or $H^{+} H^{-}$. The different types of exotic decay modes for the $2 \mathrm{HDM}$ are summarized in table 2 .

The impact of the presence of exotic Higgs decay modes on the branching ratios is shown in figure 4 for $c_{\beta-\alpha}=0$. The top two panels show the relevant branching fractions of $A$ with $m_{H}=m_{H^{ \pm}}<m_{A}$ (left), and $m_{H}<m_{A}=m_{H^{ \pm}}$(right) for $m_{A}=500 \mathrm{GeV}$ and $m_{H}=200 \mathrm{GeV}$, with $m_{12}^{2}=m_{H}^{2} s_{\beta} c_{\beta}$. In the former case, the decays $A \rightarrow H^{ \pm} W^{\mp}$ (solid blue) and $A \rightarrow H Z$ (solid green) completely dominate over the SM decays $A \rightarrow t t, b b, \tau \tau$ for most values of $t_{\beta}$, with $\operatorname{BR}\left(A \rightarrow H^{ \pm} W^{\mp}\right) \sim 50-60 \%$ and $\operatorname{BR}(A \rightarrow H Z) \sim 20-30 \%$, while in the latter with $A \rightarrow H^{ \pm} W^{\mp}$ being absent, the branching fraction of $A \rightarrow H Z$ is more than $50 \%$. Decays of $A \rightarrow t t, b b$ are only important for very small or very large $t_{\beta}$. The dashed lines show for comparison the branching fractions into the conventional SM states when the exotic decays are absent, which highlights the suppression the SM channels suffer in the presence of the exotic decays. The bottom left panel in figure 4 

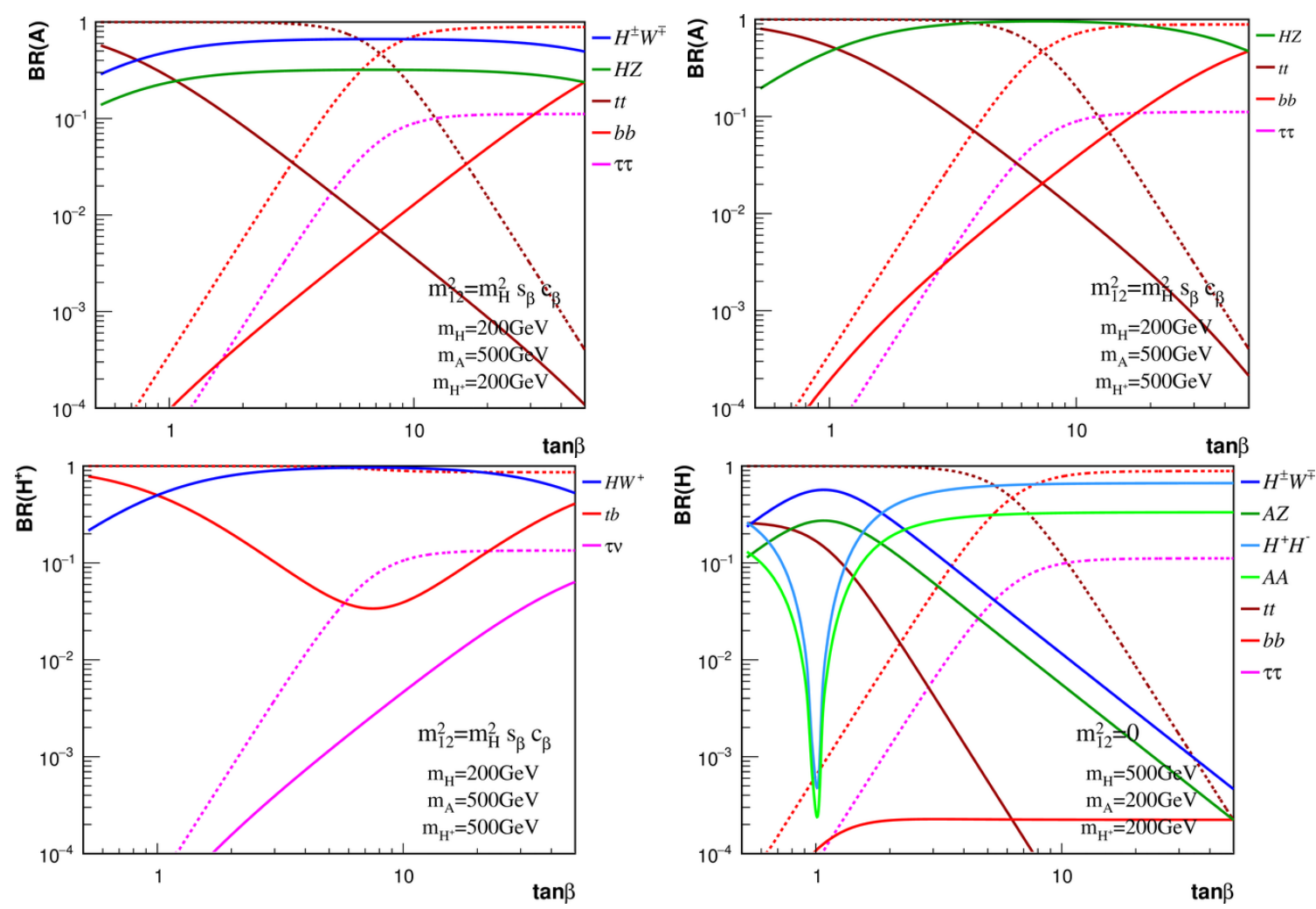

Figure 4. Branching fractions in Type II $2 \mathrm{HDM}$ as a function of $t_{\beta}$ for $c_{\beta-\alpha}=0$, with parent and daughter scalar masses fixed to $500 \mathrm{GeV}$ and $200 \mathrm{GeV}$, respectively. Top: branching fractions for $A$ with $m_{H}=m_{H^{ \pm}}<m_{A}$ (left) and with $m_{H}<m_{A}=m_{H^{ \pm}}$(right), in both cases for $m_{12}^{2}=m_{H}^{2} s_{\beta} c_{\beta}$. Bottom: branching fractions for $H^{ \pm}$with $m_{H}<m_{H^{ \pm}}=m_{A}$ and $m_{12}^{2}=m_{H}^{2} s_{\beta} c_{\beta}$ (left) and for $H$ with $m_{H}>m_{H^{ \pm}}=m_{A}$ and $m_{12}^{2}=0$ (right). In all cases, dashed lines indicate the branching fractions to SM fermion pairs when exotic decay modes are absent.

shows the branching fractions of $H^{ \pm}$for $m_{H}<m_{A}=m_{H^{ \pm}}$(with $m_{A}=500 \mathrm{GeV}$ and $m_{H}=200 \mathrm{GeV}$ ) and $m_{12}^{2}=m_{H}^{2} s_{\beta} c_{\beta}$. The decay $H^{ \pm} \rightarrow H W^{ \pm}$(solid blue) dominates with $\operatorname{BR}\left(H^{ \pm} \rightarrow H W^{ \pm}\right) \gtrsim 50 \%$, particularly for a not too heavy state $H^{ \pm}$. In that case, $H^{ \pm} \rightarrow t b$ is suppressed to be about few percent for intermediate $t_{\beta}$, and only reaches about $50 \%$ in the very small and very large $t_{\beta}$ region. Finally, the bottom right panel in figure 4 shows the branching fractions of $H$ for $m_{H}>m_{H^{ \pm}}=m_{A}$ (with $m_{H}=500 \mathrm{GeV}$ and $m_{A}=200 \mathrm{GeV}$ ) and $m_{12}^{2}=0$. In this case the decays $H \rightarrow A A$ and $H \rightarrow H^{+} H^{-}$ are allowed and dominate over most of the $t_{\beta}$ region, except for $t_{\beta} \sim 1$, where $H \rightarrow A Z$ and $H \rightarrow H^{ \pm} W^{\mp}$ become dominant due to the accidental suppression of the $\mathrm{HH}^{+} \mathrm{H}^{-}$ and $H A A$ couplings at $t_{\beta} \sim 1$. Note however that for $m_{12}^{2}=0$ the theoretical constraints do not allow a significant departure from $t_{\beta} \sim 1$, such that a large branching fraction for $H \rightarrow A Z$ and $H \rightarrow H^{ \pm} W^{\mp}$ is expected. Decays to SM fermions are highly suppressed in this scenario. 


\section{$5 \quad 2 \mathrm{HDM}$ planes for exotic Higgs decays}

Our analysis of exotic Higgs decays in the 2HDM focuses on a few key benchmark planes which show the complementarity among different LHC search channels for the new scalars. We first focus on the alignment limit: $c_{\beta-\alpha}=0$ for $m_{H}>m_{h}=125 \mathrm{GeV}$ and $s_{\beta-\alpha}=0$ for $m_{h}<m_{H}=125 \mathrm{GeV}$. In this context, we consider two possible mass planes: $m_{A}$ vs. $m_{H}=m_{H^{ \pm}}$(Plane I) and $m_{H}$ vs. $m_{A}=m_{H^{ \pm}}$(Plane II). These two choices are motivated by EWPO constraints (recall the discussion in section 3.3). This is in contrast to a potential $m_{H^{ \pm}}$vs. $m_{H}=m_{A}$ plane, highly constrained by EWPO to a small mass splitting $m_{H^{ \pm}}-$ $m_{H / A}$ which closes the phase space needed for on-shell exotic Higgs decays, ${ }^{10}$ so that we don't consider such benchmark plane in our current study. Finally let us remark that, while we do not impose the flavour bounds as hard constraints on our 2HDM benchmark planes (recall the discussion in section 3.4), we do show them as indicative in the following. Our 2HDM benchmark plane (BP) scenarios in alignment are then:

\section{Plane I: $m_{A}$ vs. $m_{H}=m_{H^{ \pm}}$.}

- BP IA: $m_{A}>m_{H}=m_{H^{ \pm}}$.

As discussed in section 3.6, this mass ordering is allowed for Case $1\left(m_{12}^{2}=m_{H}^{2} s_{\beta} c_{\beta}\right.$ and all $t_{\beta}$ values $)$ and Case $2\left(m_{12}^{2}=0\right.$ and $\left.t_{\beta} \sim 1\right)$. We thus consider four scenarios: Case 1 with $t_{\beta}=1.5,7,30$ and Case 2 with $t_{\beta}=1.5$.

- BP IB: $m_{A}<m_{H}=m_{H^{ \pm}}$.

This mass ordering is not compatible with Case 1 due to vacuum stability (see sections 3.1 and 3.6). Thus, we only consider Case 2 with $t_{\beta}=1.5 .{ }^{11}$

Plane II: $m_{H}$ vs. $m_{A}=m_{H^{ \pm}}$.

- BP IIA: $m_{H}>m_{A}=m_{H^{ \pm}}$.

As for BP IB, this mass ordering is not compatible with Case 1, and so we only consider Case 2 with $t_{\beta}=1.5$.

- BP IIB: $m_{H}<m_{A}=m_{H^{ \pm}}$.

As for BP IA, we consider Case 1 with $t_{\beta}=1.5,7,30$ and Case 2 with $t_{\beta}=1.5$.

In order to study the decays of the new scalars into the SM-like Higgs, we also consider a plane in which the departure from alignment is explored, assuming $h$ is the $125 \mathrm{GeV}$ SMlike Higgs (Plane III). We set $m_{H}=m_{A}=m_{H^{ \pm}}$for simplicity, and define the plane as $m_{H}=m_{A}=m_{H^{ \pm}}$vs. $c_{\beta-\alpha}$ :

Plane III: $m_{A}=m_{H}=m_{H^{ \pm}}$vs. $c_{\beta-\alpha}$.

\section{- BP III:}

We consider Case 1 with $t_{\beta}=1.5,7,30$ and Case 2 with $t_{\beta}=1.5$.

\footnotetext{
${ }^{10}$ As discussed in section 3.3, a sizable departure from alignment could allow for a mass hierarchy $m_{A}>$ $m_{H}+m_{Z}$ (such that $A \rightarrow H Z$ is kinematically allowed) and $m_{H} \gtrsim m_{H^{ \pm}}+m_{W}$ (such that $H \rightarrow H^{ \pm} W^{\mp}$ is kinematically allowed, but nevertheless phase space suppressed). The phenomenology of this kind of scenario is however largely contained in Planes I-II, and so we do not consider it separately.

${ }^{11} t_{\beta} \neq 1$ is chosen for the exotic decays into two lighter new scalars ( $H \rightarrow A A$ in this case) not to vanish.
} 


\begin{tabular}{|c|c|c|c|c|c|}
\hline & Mass Planes & decays & $m_{12}^{2}$ & $\tan \beta$ & Figures \\
\hline \multirow[t]{2}{*}{ BP IA } & \multirow{2}{*}{$m_{A}>m_{H}=m_{H^{ \pm}}$} & \multirow{2}{*}{$\begin{array}{c}A \rightarrow H^{ \pm} W^{\mp} \\
A \rightarrow H Z\end{array}$} & $m_{H}^{2} s_{\beta} c_{\beta}$ & $1.5,7,30$ & \multirow[t]{2}{*}{5,6} \\
\hline & & & 0 & 1.5 & \\
\hline BP IB & $m_{A}<m_{H}=m_{H^{ \pm}}$ & $\begin{array}{c}H \rightarrow A Z, H \rightarrow A A \\
H^{ \pm} \rightarrow A W^{ \pm}\end{array}$ & 0 & 1.5 & 9 \\
\hline BP IIA & $m_{H}>m_{A}=m_{H^{ \pm}}$ & $\begin{aligned} H \rightarrow A Z, H & \rightarrow A A \\
H \rightarrow H^{+} H^{-}, H & \rightarrow H^{ \pm} W^{\mp}\end{aligned}$ & 0 & 1.5 & 10 \\
\hline \multirow[t]{2}{*}{ BP IIB } & \multirow[t]{2}{*}{$m_{H}<m_{A}=m_{H^{ \pm}}$} & \multirow{2}{*}{$\begin{array}{c}A \rightarrow H Z \\
H^{ \pm} \rightarrow H W^{ \pm}\end{array}$} & $m_{H}^{2} s_{\beta} c_{\beta}$ & $1.5,7,30$ & \multirow[t]{2}{*}{7,8} \\
\hline & & & 0 & 1.5 & \\
\hline \multirow[t]{2}{*}{ BP III } & \multirow{2}{*}{$\begin{array}{c}m_{A}=m_{H}=m_{H^{ \pm}} \\
\quad \text { vs. } c_{\beta-\alpha}\end{array}$} & \multirow{2}{*}{$\begin{array}{c}A \rightarrow h Z, H^{ \pm} \rightarrow h W^{ \pm} \\
H \rightarrow h h\end{array}$} & $m_{H}^{2} s_{\beta} c_{\beta}$ & $1.5,7,30$ & \multirow[t]{2}{*}{$11,12,13$} \\
\hline & & & 0 & 1.5 & \\
\hline
\end{tabular}

Table 3. Summary table of the different 2HDM benchmark planes.

A summary of the different benchmark planes considered and the relevant exotic decay modes is shown in table 3. In all cases, we present the $\sigma \times \mathrm{BR}$ of each characteristic decay channel at the $13 \mathrm{TeV}$ LHC, together with a detailed analysis of the regions disfavoured by theoretical and experimental constraints (including flavour constraints, shown for reference only). The results for Planes I and II $\left(c_{\beta-\alpha}=0\right)$ are presented in section 5.1, while the results for decays to SM-like Higgs away from alignment, corresponding to Plane III, are presented in section 5.2. Further details on the cross sections and decay branching fractions for the non-SM like Higgses can be found in appendix A.

Before we move on to discuss in detail our different 2HDM planes for LHC searches at $13 \mathrm{TeV}$, let us comment on the comparison of these benchmark scenarios with others proposed in the literature. In particular, our Planes I and II have a substantial overlap with the $2 \mathrm{HDM}$ "short cascade" scenario D from [71], while our specific BP IA and BP IIB have similarities with the $A \rightarrow H Z$ benchmarks for $c_{\beta-\alpha}=0$ in [19] (see also [22]). As compared to [71], the present analysis explores the full mass plane, not restricted to specific benchmark lines with fixed relations ${ }^{12}$ among $m_{H}, m_{A}$ and $m_{H^{ \pm}}$. We also explore the dependence on $t_{\beta}$, which has a significant impact on the allowed $2 \mathrm{HDM}$ parameter space for Planes I and II. Moreover, our analysis includes the $8 \mathrm{TeV}$ experimental constraint from the CMS $H \rightarrow A Z / A \rightarrow H Z$ search [26, 27], precisely tailored to probe these $2 \mathrm{HDM}$ scenarios and thus a key ingredient in a study of 2HDM exotic Higgs decays.

It is worth discussing here the extent to which a departure from our Benchmark Plane assumptions (Case 1 with $m_{12}^{2}=m_{H}^{2} s_{\beta} c_{\beta}$ or Case 2 with $m_{12}^{2}=0$, with certain mass degeneracy relations between $m_{A}, m_{H}$ and $m_{H^{ \pm}}$) may lead to modifications of the 2HDM phenomenology w.r.t. the scenarios we consider in this work:

- Negative $m_{12}^{2}$. Extending $m_{12}^{2}$ to be negative does not introduce further constraints regarding vacuum stability. In addition, the variation of unitarity constraints for

\footnotetext{
${ }^{12}$ In particular, we note that the fixed relations in [71] result in the exotic Higgs decays being largely subdominant above the $t \bar{t}$ threshold, which may not be the case in general (see e.g. figure 4 ).
} 
$m_{12}^{2}<0$ w.r.t. the $m_{12}^{2}=0$ case is mild, such that $m_{12}^{2}=0$ effectively captures this scenario.

- Deviation from $m_{12}^{2}=m_{H}^{2} s_{\beta} c_{\beta}$. Assuming the alignment limit $c_{\beta-\alpha}=0$, at high $t_{\beta}$ the soft $\mathbb{Z}_{2}$ breaking term $m_{12}^{2}$ is fixed to be around $m_{H}^{2} s_{\beta} c_{\beta}$ by unitarity. Only when departing significantly from the alignment limit are deviations from $m_{12}^{2}=m_{H}^{2} s_{\beta} c_{\beta}$ at high $t_{\beta}$ possible, yielding a larger allowed region in parameter space.

At low values of $t_{\beta}$ deviations from $m_{12}^{2}=m_{H}^{2} s_{\beta} c_{\beta}$ can be easily accommodated in the alignment limit. We however stress that changing the value of $m_{12}^{2}$ only affects cubic and quartic Higgs couplings. In particular, $H^{+} H^{-}$and $H A A$ coupling vanish for $m_{12}^{2}=m_{H}^{2} s_{\beta} c_{\beta}$ in the alignment limit (see section 3.6). Deviations from $m_{12}^{2}=$ $m_{H}^{2} s_{\beta} c_{\beta}$ will then increase the branching fractions of $H \rightarrow A A$ and $H \rightarrow H^{+} H^{-}$(if allowed by kinematics) with the branching fractions to other final states decreasing accordingly. For $m_{12}^{2}$ far away from $m_{H}^{2} s_{\beta} c_{\beta}$, the branching fraction dependence on $m_{12}^{2}$ is mild, with Case $2\left(m_{12}^{2}=0\right)$ being a representative scenario.

- Deviations from the alignment limit $c_{\beta-\alpha}=0$. In our discussion we mainly focus on the alignment limit, preferred by Higgs coupling measurements at the LHC. In particular, we find that in the alignment limit, unitarity and vacuum stability cannot be satisfied simultaneously if $m_{H}>m_{A}$ or $m_{H^{ \pm}}$at high $t_{\beta}$ and therefore decays of the type $H \rightarrow A Z, H \rightarrow H^{ \pm} W^{\mp}$ are not permitted for large $t_{\beta}$. This statement can be relaxed when deviating from the alignment limit. For moderate values of $\left|c_{\beta-\alpha}\right|$ around $0.2-0.6$, there are regions of parameter space with $m_{H}>m_{A}, m_{H^{ \pm}}$, which are allowed by both unitarity and vacuum stability. Note however that once away from the alignment limit, channels like $H \rightarrow A Z, H^{ \pm} W^{\mp}$ will generically have reduced branching fractions. There have been studies in the literature for exotic Higgs decays in those non-alignment region [22, 72, 73].

- Deviation from mass degeneracy. The mass degeneracy relation is mainly imposed by the electroweak precision measurements. As shown in the left panel of figure 2, small deviations from $m_{H^{ \pm}} \approx m_{A / H}$ are allowed. In principle, there are small regions of parameter space corresponding to a mass hierarchy $m_{H}=m_{A}<m_{H^{ \pm}}$. However, these region of parameter space are already in almost $2 \sigma$ tension with observation. Other deviations from mass degeneracy will not lead to a phenomenology that would differ significantly from that of BP I and BP II.

\subsection{Exotic decays in the alignment limit}

\subsubsection{BP IA: $m_{A}>m_{H}=m_{H^{ \pm}}$}

In this scenario, and for a sufficient mass splitting, there are two dominant exotic decay channels: $A \rightarrow H^{ \pm} W^{\mp}$ and $A \rightarrow H Z$, for which we respectively show the $\sigma \times \mathrm{BR}$ in figures 5 and 6 . In each case, we show four panels corresponding to the choices of $m_{12}^{2}$ and $t_{\beta}$ described in table 3 . Note that for $t_{\beta}=1.5,7$ we consider the dominant $g g A$ production, while for $t_{\beta}=30$ the $b b A$ production dominates and is considered instead. For each panel, 

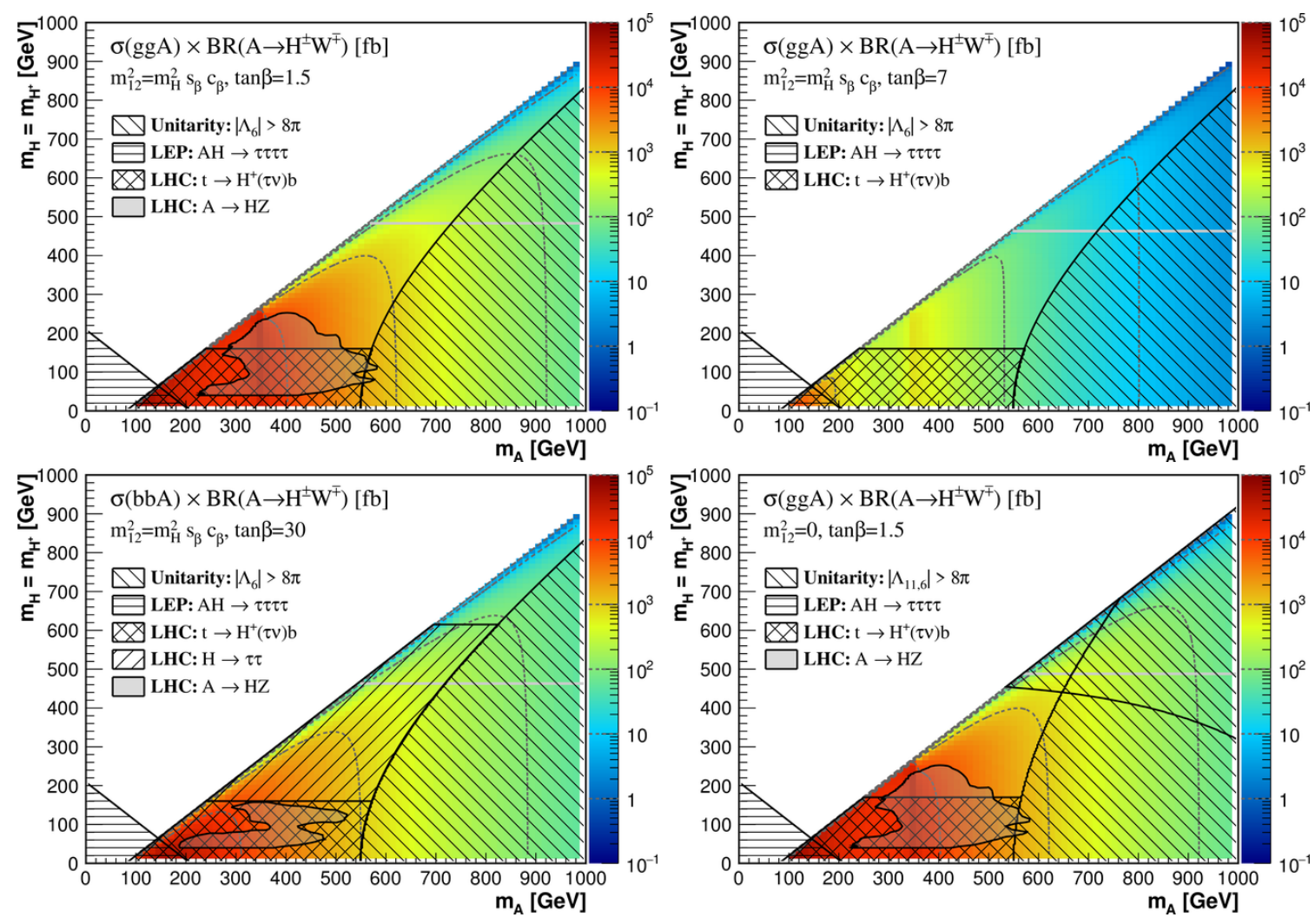

Figure 5. $\sigma \times$ BR for the exotic decay $A \rightarrow H^{ \pm} W^{\mp}$ in BP IA: $m_{A}$ vs $m_{H}=m_{H^{ \pm}}$plane, for Case 1 with $t_{\beta}=1.5$ (upper left), 7 (upper right), 30 (lower left) and Case 2 with $t_{\beta}=1.5$ (lower right). Contour lines of $10,10^{2}, 10^{3}$ and $10^{4} \mathrm{fb}$ are drawn as light grey dashed curves to guide the eye. The shaded areas enclosed by an irregular curve and hatched regions are ruled out by theoretical and experimental constraints (see text for details). The solid horizontal grey line indicates the flavour constraint $m_{H^{ \pm}}>480 \mathrm{GeV}$.

contour lines of $10,10^{2}, 10^{3}$ and $10^{4} \mathrm{fb}$ are drawn as light grey dashed curves to guide the eye. Large cross sections $\sigma \times \mathrm{BR} \gtrsim 1 \mathrm{pb}$ are possible for $t_{\beta} \sim 1$ and $t_{\beta} \gg 1$, respectively due to the enhanced top and bottom Yukawa coupling contribution, even for large CP-odd scalar masses $m_{A} \sim 500-600 \mathrm{GeV}$. The shaded areas enclosed by an irregular curve in figures 5 and 6 are excluded by the CMS $A \rightarrow H Z$ search [26, 27], which already constrains a sizable portion of parameter space and highlights the potential of such a search at LHC $13 \mathrm{TeV}$ in the bbll and $\tau \tau \ell \ell$ final states, as a probe of both $A$ and $H$.

Hatched regions show the parameter space excluded by other experimental searches, as well as unitarity constraints. The former exclusions are mainly due to $t \rightarrow H^{+} b$ searches, which yield a limit $m_{H^{ \pm}}>m_{t}$, as well as $H \rightarrow \tau \tau$ searches for large $t_{\beta}$, which rule out $m_{H}<600 \mathrm{GeV}$ for $t_{\beta}=30$. We also show the flavour bound $m_{H^{ \pm}}>480 \mathrm{GeV}$ as a horizontal grey line for indicative purposes.

Regarding unitarity, for Case $1\left(m_{12}^{2}=m_{H}^{2} s_{\beta} c_{\beta}\right)$ with $m_{A}>m_{H}=m_{H^{ \pm}}$the eigenvalues of the scattering matrix are $\left|\Lambda_{i \neq 6}\right| v^{2}=m_{A}^{2}-m_{H}^{2} \pm \mathcal{O}\left(m_{h}^{2}\right)$ and $\left|\Lambda_{6}\right| v^{2}=$ $5\left(m_{A}^{2}-m_{H}^{2}\right)+m_{h}^{2}$. The latter imposes the strongest constraint, which rules out regions with a very large mass splitting $m_{A}-m_{H}$ (as indicated by the hatched region in the lower- 



Figure 6. $\sigma \times \mathrm{BR}$ for the exotic decay $A \rightarrow H Z$ in BP IA: $m_{A}$ vs $m_{H}=m_{H^{ \pm}}$plane (see caption of figure 5 for further details). For $t_{\beta}=1.5$, the contour line at $\sigma \times \mathrm{BR}=10 \mathrm{pb}$ around $m_{A}=350 \mathrm{GeV}$ is caused by the enhanced $g g \rightarrow A$ production cross section at the top threshold.

right corner of each panel in figures 5 and 6$)$. For Case $2\left(m_{12}^{2}=0\right)$ with $m_{A}>m_{H}=$ $m_{H^{+}}$, the strongest unitarity constraints come from $\left|\Lambda_{6}\right| v^{2}=5 m_{A}^{2}-3 m_{H}^{2} \pm \mathcal{O}\left(m_{h}^{2}\right)$ and $\left|\Lambda_{11}\right| v^{2}=\frac{1}{2} m_{H}^{2}\left(\frac{1}{t_{\beta}^{2}}+t_{\beta}^{2}\right)+\frac{1}{2} \sqrt{9 m_{H}^{4}\left(\frac{1}{t_{\beta}^{2}}-t_{\beta}^{2}\right)^{2}+4 m_{A}^{4}} \pm \mathcal{O}\left(m_{h}^{2}\right)$. In particular, $\left|\Lambda_{11}\right|$ rules out the large $m_{H}$ region (upper hatched region in the lower right panel in figures 5 and 6 ).

Taking into account both the theoretical and experimental constraints, relatively large regions of $m_{A}$ vs. $m_{H}=m_{H^{ \pm}}$remain viable and having a sizable signal cross section for small to intermediate values of $t_{\beta}$ for Case I. For $t_{\beta} \gg 1$, only the region $m_{A} \gtrsim$ $m_{H}=m_{H^{ \pm}}>600 \mathrm{GeV}$ still survives. For Case 2, given the unitary constraints ruling out large values of $m_{H}$ and $m_{A}$, only the region $200 \mathrm{GeV}<m_{A}<650 \mathrm{GeV}$ and $175 \mathrm{GeV}$ $<m_{H}=m_{H^{ \pm}}<450 \mathrm{GeV}$ remains viable.

\subsubsection{BP IIB: $m_{H}<m_{A}=m_{H^{ \pm}}$}

For $m_{H}<m_{A}=m_{H^{ \pm}}$, the dominant exotic decay channels are $A \rightarrow H Z$ and $H^{ \pm} \rightarrow H W^{ \pm}$. We show the $\sigma \times \mathrm{BR}$ for $A \rightarrow H Z$ and $H^{ \pm} \rightarrow H W^{ \pm}$respectively in figures 7 and 8 . The low $m_{A}+m_{H}$ region is ruled out by the LEP search $e^{+} e^{-} \rightarrow A H$ (recall the discussion in section 3.5), while unitarity constraints bound large values for $m_{A}, m_{H}$ : for Case I the strongest constraints arise from $\left|\Lambda_{2,4,5,6,11,12}\right| v^{2}=3\left(m_{A}^{2}-m_{H}^{2}\right) \pm \mathcal{O}\left(m_{h}^{2}\right)$, which limit $m_{A}^{2}-m_{H}^{2}$ for large $m_{H}$ and/or $m_{A}$. For Case 2, large values of either $m_{A}$ or $m_{H}$ are 

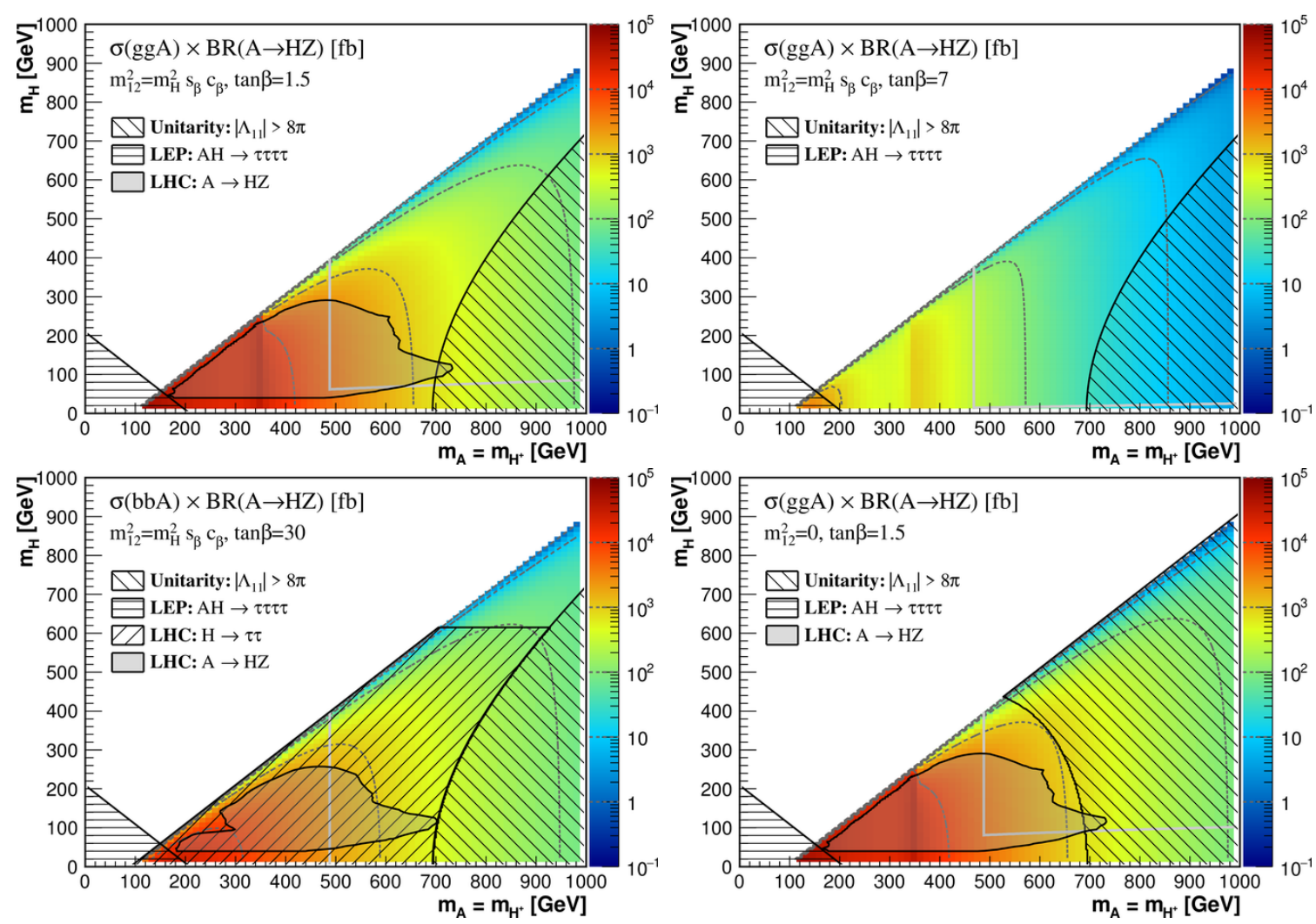

Figure 7. $\sigma \times$ BR for the exotic decay $A \rightarrow H Z$ in BP IIB: $m_{A}=m_{H^{ \pm}}$vs $m_{H}$ plane (see caption of figure 5 for further details). The solid horizontal and vertical light grey lines indicate the various flavour constraints.

excluded, since the strongest unitarity constraint comes from $\left|\Lambda_{11}\right| v^{2}=\frac{1}{2} m_{H}^{2}\left(\frac{1}{t_{\beta}^{2}}+t_{\beta}^{2}\right)+$ $\frac{1}{2} \sqrt{9 m_{H}^{4}\left(\frac{1}{t_{\beta}^{2}}-t_{\beta}^{2}\right)^{2}+4\left(3 m_{A}^{2}-2 m_{H}^{2}\right)^{2}} \pm \mathcal{O}\left(m_{h}^{2}\right)$.

For Case 1 with $t_{\beta}=1.5$ (upper left panel of figure 7 ), signal cross sections for $A \rightarrow H Z$ in excess of $10 \mathrm{pb}$ are viable given all the constraints, while we note that the LHC Run 1 CMS $A \rightarrow H Z$ search rules out a large portion of the parameter space with $m_{H}<300 \mathrm{GeV}$ and $m_{A}<650 \mathrm{GeV}$. Intermediate values of $t_{\beta}$ (exemplified by the $t_{\beta}=7$ case shown in the upper right panel of figure 7) only permit signal cross sections below $1 \mathrm{pb}$, due to the small gluon fusion production cross section (for $m_{A}>600 \mathrm{GeV}$ the $\sigma \times$ BR values are in fact below $20 \mathrm{fb}$ ). For $t_{\beta}=30$ (lower left panel of figure 7 ), the current collider search of $H \rightarrow \tau \tau$ rules out $m_{H}<600 \mathrm{GeV}$, leaving only a small corner of parameter space allowed, with signal cross sections $\sigma \times \mathrm{BR} \lesssim 100 \mathrm{fb}$. For Case 2 , the lower right panel of figure 7 shows that the CMS $A \rightarrow H Z$ search constrains most of the viable parameter space, which may in turn be probed completely by LHC 13 .

While the generic features for $H^{ \pm} \rightarrow H W^{ \pm}$are similar to those of $A \rightarrow H Z$, the signal cross sections are about two order of magnitude smaller, due to the suppressed production cross section of $p p \rightarrow H^{ \pm} t b$. This, in addition to the complicated final state $H W^{+} W^{-} b b$ which results, makes this channel challenging for LHC studies at $13 \mathrm{TeV}$. 

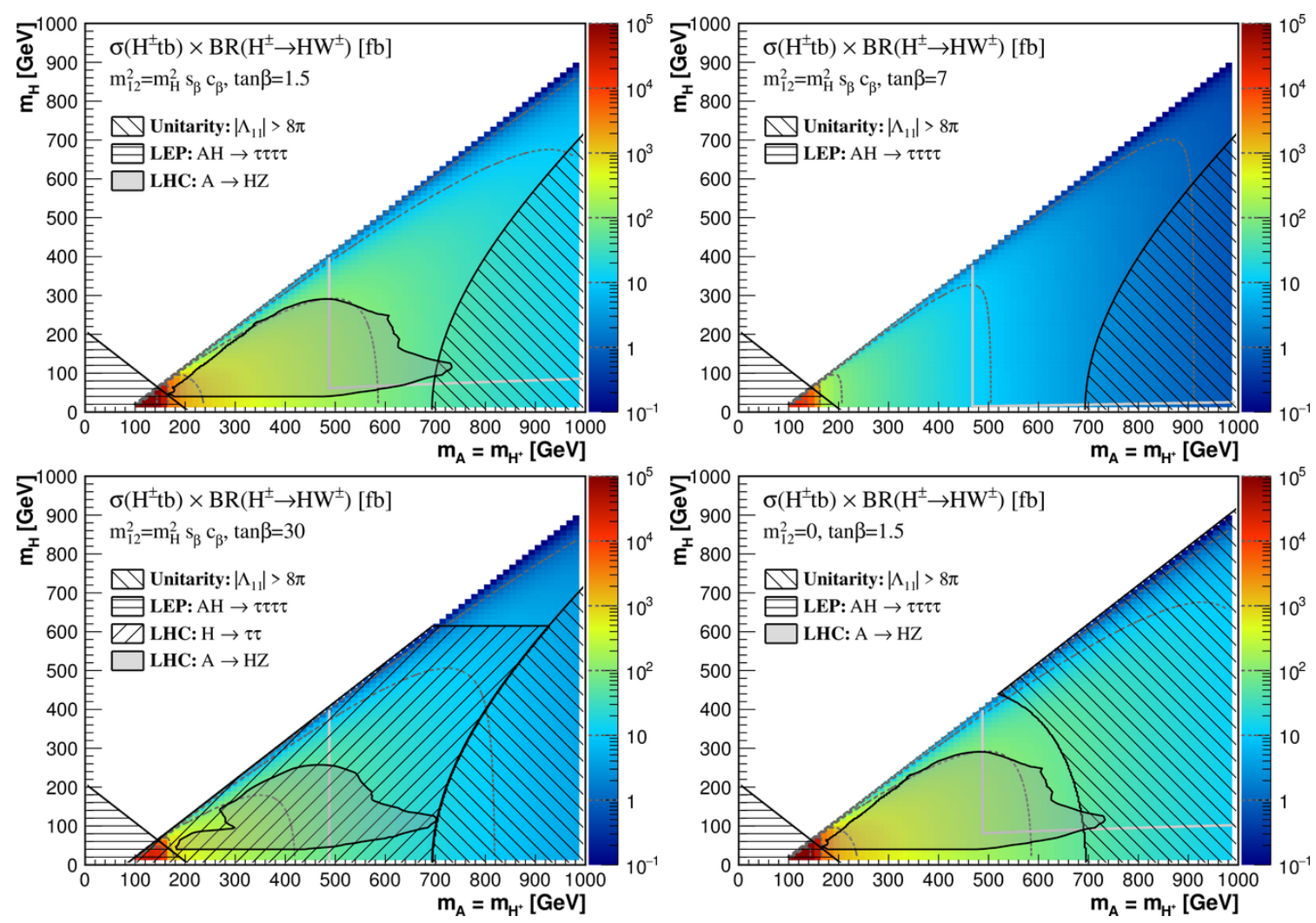

Figure 8. $\sigma \times$ BR for the exotic decay $H^{ \pm} \rightarrow H W^{ \pm}$in BP IIB: $m_{A}=m_{H^{ \pm}}$vs. $m_{H}$ plane (see caption of figure 5 for further details). The solid horizontal and vertical light grey lines indicate the various flavour constraints.

\subsubsection{BP IB: $m_{A}<m_{H^{ \pm}}=m_{H}$}

In this scenario, only Case $2\left(m_{12}^{2}=0\right)$ is viable. The $\sigma \times \mathrm{BR}$ for the three possible exotic decay channels $H \rightarrow A Z, H^{ \pm} \rightarrow A W^{ \pm}$and $H \rightarrow A A$ is shown in figure 9 for our benchmark $t_{\beta}=1.5$. The $m_{H}>460 \mathrm{GeV}$ region is excluded by unitarity, the strongest unitarity constraint coming from $\left|\Lambda_{11}\right| v^{2}=\frac{1}{2} m_{H}^{2}\left(\frac{1}{t_{\beta}^{2}}+t_{\beta}^{2}\right)+\frac{1}{2} \sqrt{9 m_{H}^{4}\left(\frac{1}{t_{\beta}^{2}}-t_{\beta}^{2}\right)^{2}+4 m_{A}^{4}} \pm \mathcal{O}\left(m_{h}^{2}\right)$.

Below the unitarity limit on $m_{H}$, the ATLAS/CMS limits on $A \rightarrow \tau \tau$ at low $t_{\beta}(g g A$ production) $[50,51]$ combined with the bounds from the CMS $H \rightarrow A Z$ search $[26,27]$ rule out $m_{A}>40 \mathrm{GeV}$ down to $m_{H} \lesssim 350 \mathrm{GeV}$. As can be seen from figure 9 , only a small region of parameter space survives the unitarity and $\mathrm{LHC} 8 \mathrm{TeV}$ constraints. We also stress that in this case including the flavour constraint $m_{H^{ \pm}}>480 \mathrm{GeV}$ would rule out this benchmark scenario completely.

\subsubsection{BP IIA: $m_{H}>m_{A}=m_{H^{ \pm}}$}

Four exotic Higgs decay channels, $H \rightarrow A Z, H \rightarrow H^{ \pm} W^{\mp}, H \rightarrow A A$, and $H \rightarrow H^{+} H^{-}$are possible for BP IIA (which we recall is only allowed for Case 2), shown respectively in the four panels of figure 10. Comparing to BP IIB, the additional collider search limit $m_{H^{ \pm}}>$ $m_{t}$ applies, which overlaps with the $8 \mathrm{TeV}$ LHC exclusion from $A \rightarrow \tau \tau$. This results in only a small stripe in parameter space, corresponding to $200 \mathrm{GeV}<m_{A}=m_{H^{ \pm}}<240 \mathrm{GeV}$ 


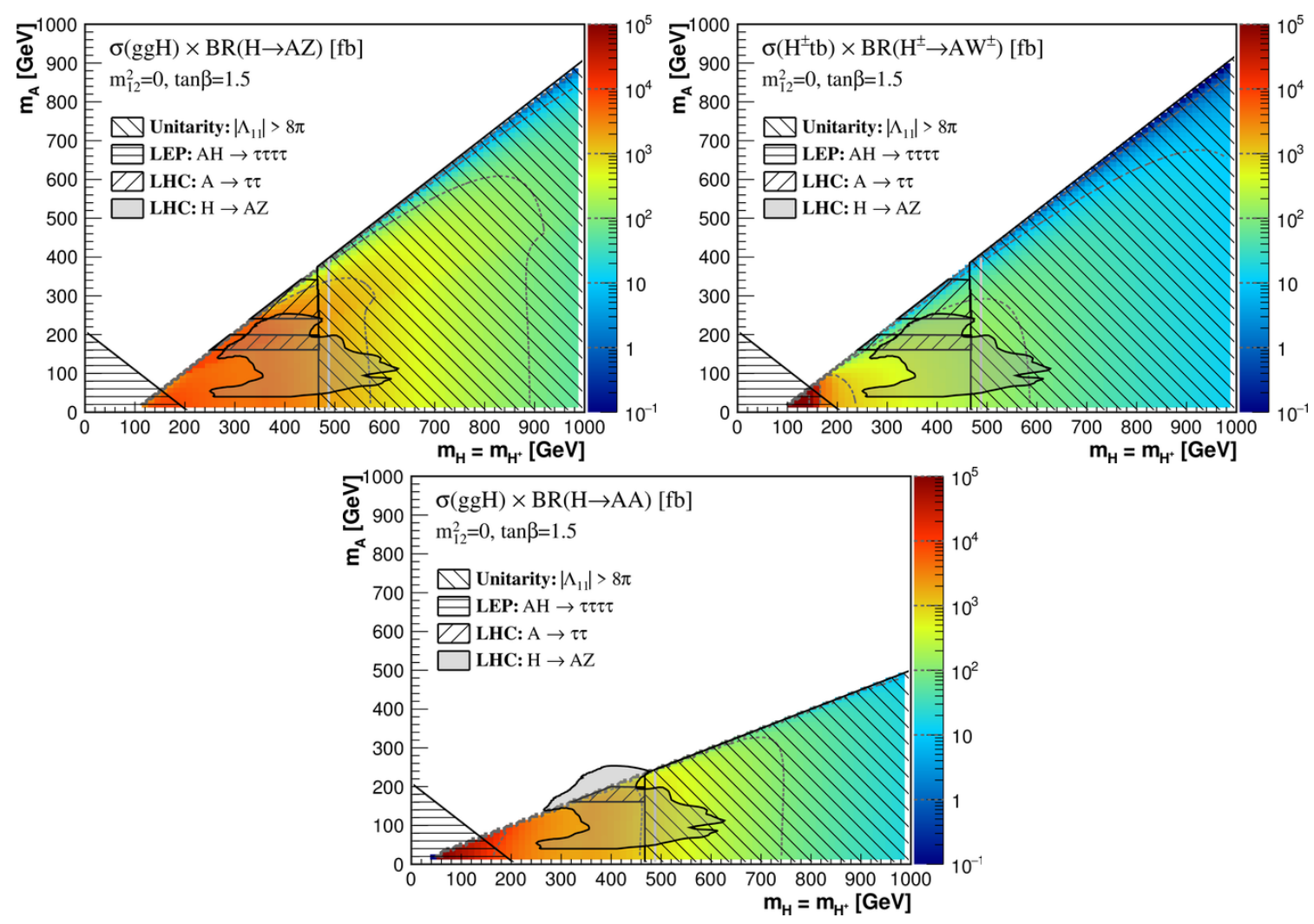

Figure 9. $\sigma \times \mathrm{BR}$ for the exotic decays $H \rightarrow A Z$ (up left), $H^{ \pm} \rightarrow A W^{ \pm}$(up right) and $H \rightarrow A A$ (down) in BP IB: $m_{H}=m_{H^{ \pm}}$vs. $m_{A}$ plane, for Case $2\left(m_{12}^{2}=0\right)$ with $t_{\beta}=1.5$. Contour lines of $10,10^{2}$ and $10^{3} \mathrm{fb}$ are drawn as light grey dashed curves to guide the eye. The shaded areas enclosed by an irregular curve and hatched regions are ruled out by theoretical and experimental constraints (see text for details). The solid (vertical) light grey lines indicate the flavour constraint $m_{H^{ \pm}}>480 \mathrm{GeV}$.

and $300 \mathrm{GeV}<m_{H}<450 \mathrm{GeV}$, being viable. Moreover, we note that the decays $H \rightarrow A A$ and $H \rightarrow H^{+} H^{-}$are essentially not kinematically allowed in the viable region, as shown in the lower panels of figure 10. This benchmark scenario should indeed be possible to probe completely at LHC $13 \mathrm{TeV}$.

\subsection{Exotic decays into $h$ away from alignment}

\subsubsection{BP III: $m_{A}=m_{H}=m_{H^{ \pm}}$vs. $c_{\beta-\alpha}$}

Exotic decays with the SM-like Higgs $h$ in the final state are possible away from the alignment limit $c_{\beta-\alpha}=0$, as the $A h Z, H^{ \pm} h W^{\mp}$ and $H h h$ couplings are proportional to $c_{\beta-\alpha}$. In figures 11, 12, and 13 we respectively show the $\sigma \times \mathrm{BR}$ for $A \rightarrow h Z, H^{ \pm} \rightarrow h W^{ \pm}$ and $H \rightarrow h h$, in each case for Case 1 with $t_{\beta}=1.5,7,30$ and Case 2 with $t_{\beta}=1.5$ in the $\left(c_{\beta-\alpha}\right.$ vs $\left.m_{A}=m_{H}=m_{H^{ \pm}}\right)$plane.

For Case 1 with $t_{\beta}=1.5$, only the region $\left|c_{\beta-\alpha}\right| \lesssim 0.2$ (close to the alignment limit) is viable as a result of Higgs signal strength measurements (mainly driven by the $g_{h V V}$ couplings) considering all the theoretical and experimental constraints. The allowed range 

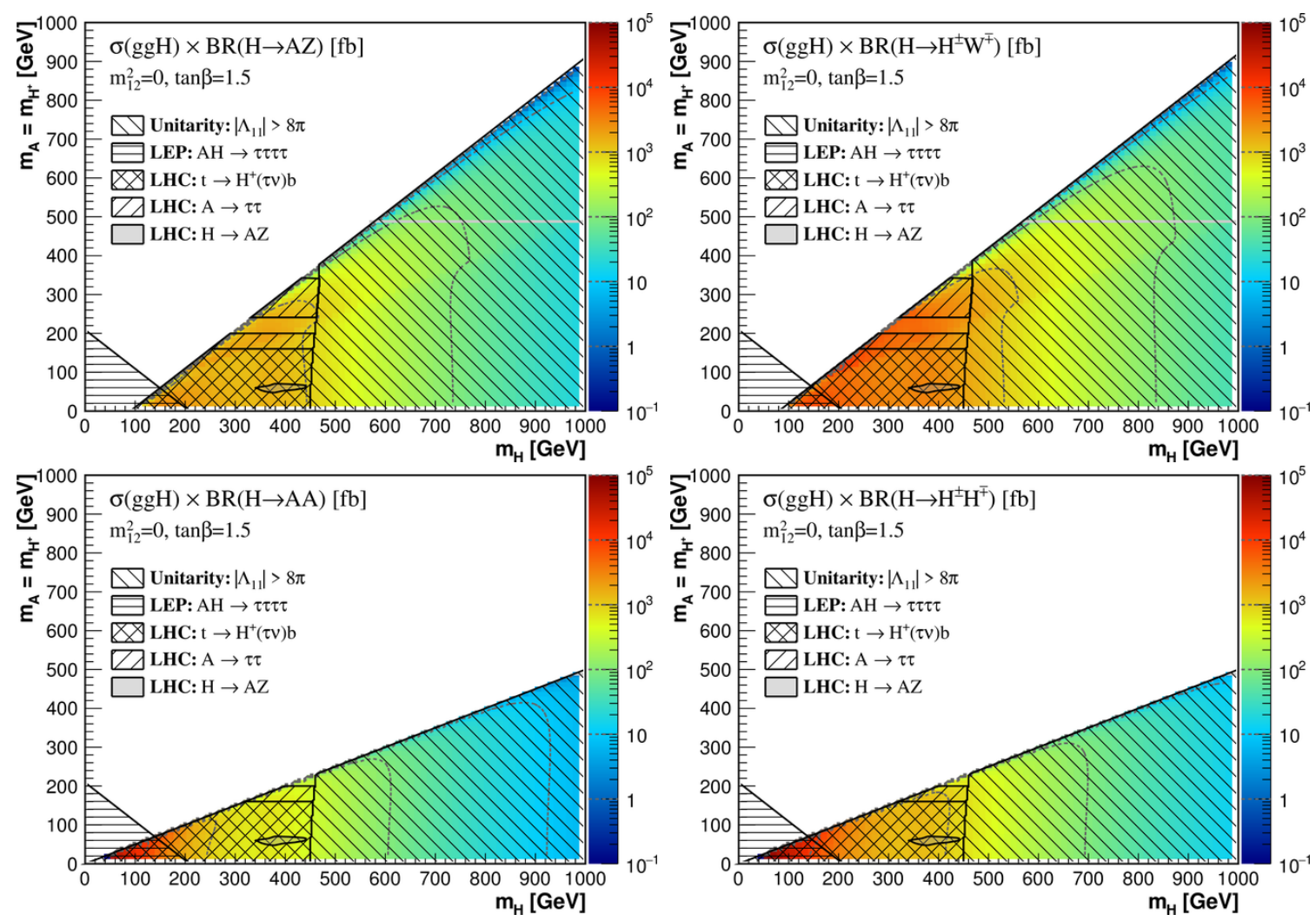

Figure 10. $\sigma \times$ BR for the exotic decays $H \rightarrow A Z$ (up left), $H^{ \pm} \rightarrow H^{ \pm} W^{\mp}$ (up right), $H \rightarrow A A$ (down left) and $H \rightarrow H^{+} H^{-}$(down right) in BP IIA: $m_{H}$ vs. $m_{A}=m_{H^{ \pm}}$plane (see caption of figure 9 for further details).

for $c_{\beta-\alpha}$ shrinks as the masses of the heavy $2 \mathrm{HDM}$ scalars grow due to stability constraints, being already restricted to $-0.02<c_{\beta-\alpha}<0.06$ for $m_{A}=m_{H}=m_{H^{ \pm}} \simeq 500 \mathrm{GeV}$. At the same time, LHC bounds on $H \rightarrow Z Z$ and $A \rightarrow \tau \tau$ rule out $m_{A}=m_{H}=m_{H^{ \pm}}<350 \mathrm{GeV}$. For significantly higher values of $t_{\beta}$ (as our $t_{\beta}=7,30$ scenarios) the stability constraints rule out almost completely the region $c_{\beta-\alpha}<0$, while unitarity imposes a strong constraint on $c_{\beta-\alpha}>0$ for high scalar masses $m_{A}=m_{H}=m_{H^{ \pm}}>600 \mathrm{GeV}$. In addition, for $t_{\beta}=7$ the vacuum stability constraint rules out the region $c_{\beta-\alpha}>0.3$ while Higgs signal strengths rule out the region $0.05<c_{\beta-\alpha}<0.24$. For $t_{\beta}=30$, Higgs signal strengths rule out $c_{\beta-\alpha} \gtrsim 0.01$, while $A \rightarrow \tau \tau$ searches restrict the allowed parameter space to $m_{A}=m_{H}=m_{H^{ \pm}}>650 \mathrm{GeV}$, leaving only a very narrow stripe as viable parameter space. For Case 2 with $t_{\beta}=1.5$, satisfying the constraints from $H \rightarrow Z Z$ and $A \rightarrow \tau \tau$ requires $m_{A}=m_{H}=m_{H^{ \pm}}>350 \mathrm{GeV}$ and $\left|c_{\beta-\alpha}\right| \lesssim 0.2$, while unitarity imposes an upper bound on the scalar masses in the range $450 \mathrm{GeV}-550 \mathrm{GeV}$ depending on $c_{\beta-\alpha}$. As shown in figure 11, the cross sections for $A \rightarrow h Z$ in the allowed region of parameter space could reach $1 \mathrm{pb}$ or higher for $t_{\beta}=1.5$ both in Case 1 and 2 . For $t_{\beta}=7$ (Case 1 ) the cross section for $A \rightarrow h Z$ is still sizable in the allowed region $0.24<c_{\beta-\alpha}<0.3$, reaching values $\sim 100 \mathrm{fb}$. For $t_{\beta}=30$ the signal cross section is however very small due to the suppressed branching ratio $\mathrm{BR}(A \rightarrow h Z)$ close to the alignment limit. The signal cross sections for $H^{ \pm} \rightarrow h W^{ \pm}$ 

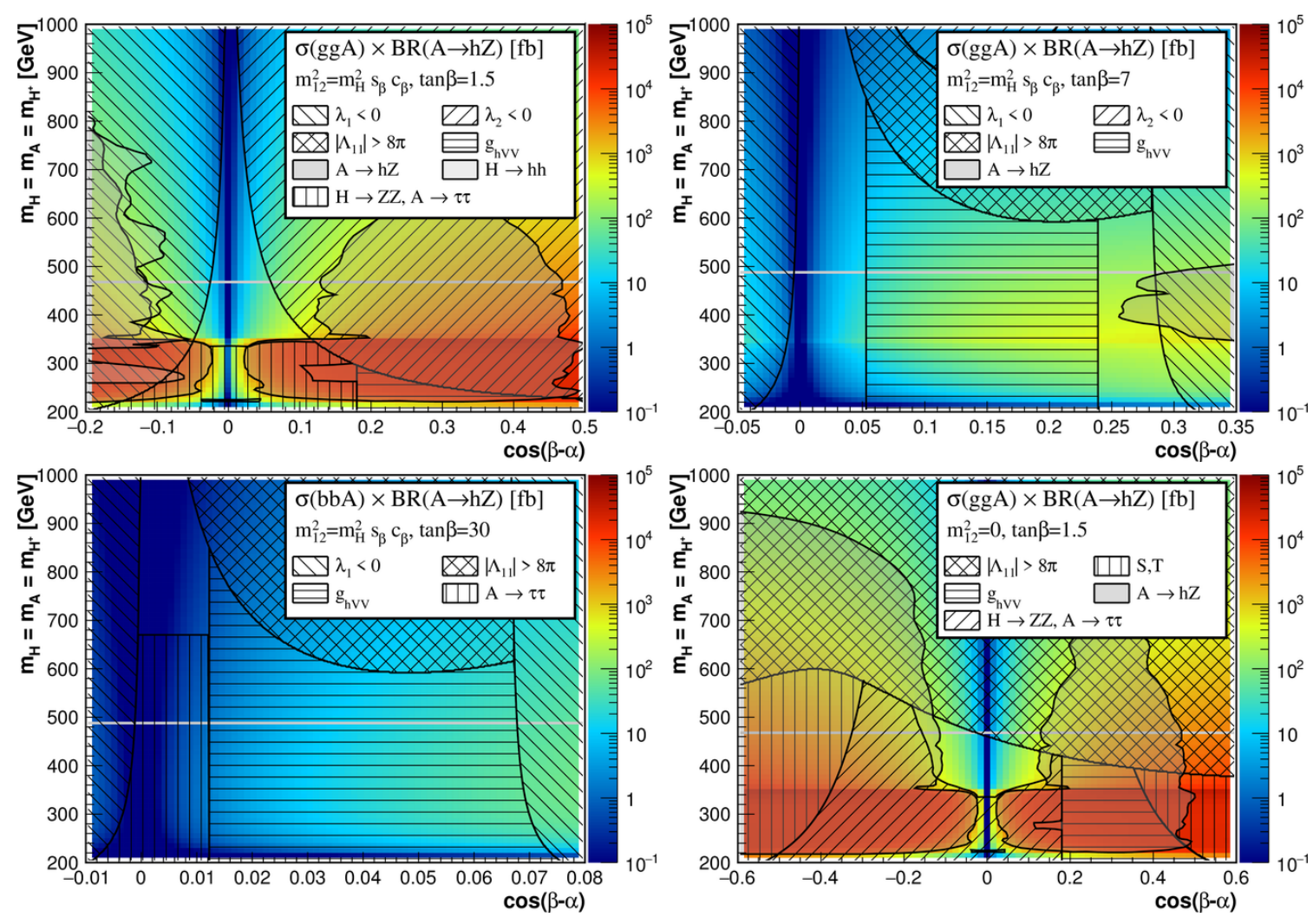

Figure 11. $\sigma \times \mathrm{BR}$ for $A \rightarrow h Z$ for the gluon fusion production in Case $1, \tan \beta=1.5$ (upper left), 7 (upper right), as well as Case $2, \tan \beta=1.5$ (lower right), and $b b A$ associated production for Case $1, \tan \beta=30$ (lower left) in BPIII: $c_{\beta-\alpha}$ vs. $m_{A}=m_{H}=m_{H^{ \pm}}$. Hatched regions are excluded by either theoretical or experimental constraints (as indicated in the legend), while the shaded areas enclosed by an irregular curve indicate the parameter space constrained by LHC searches for exotic (non-SM) Higgs decays: $A \rightarrow h Z$ and $H \rightarrow h h$. The solid horizontal light grey lines indicate the flavour constraint $m_{H^{ \pm}}>480 \mathrm{GeV}$.

shown in figure 12 follow a trend similar to those for $A \rightarrow h Z$, but being typically a factor 10-100 smaller due to the suppressed production cross section for $H^{ \pm}$above $m_{t}$ (see appendix A.1). Finally for $H \rightarrow h h$ the signal cross sections, shown in figure 13, are about factor of 10 smaller than those of $A \rightarrow h Z$, and an additional suppression of the branching ratio $\operatorname{BR}(H \rightarrow h h)$ occurs for certain values of $c_{\beta-\alpha}$ (e.g. $c_{\beta-\alpha} \sim 0.22$ for $t_{\beta}=7$ and $c_{\beta-\alpha} \sim 0.052$ for $t_{\beta}=30$, as seen from figure 13).

\section{Conclusions}

In the 2HDM, other than decaying to pairs of SM quarks, leptons, and gauge bosons, the exotic decays of heavy Higgses into two lighter Higgses or one light Higgs and a SM gauge boson are likely to dominate once they are kinematically open. While the collider search bounds for heavy Higgses based on conventional search modes $W W, Z Z, \gamma \gamma, b b$ and $\tau \tau$ for neutral Higgses, and $\tau \nu$ and $c s$ modes for charged Higgses would be relaxed once those exotic modes are open, the exotic decay modes offer new discovery channels in large regions of the 2HDM parameter space. 

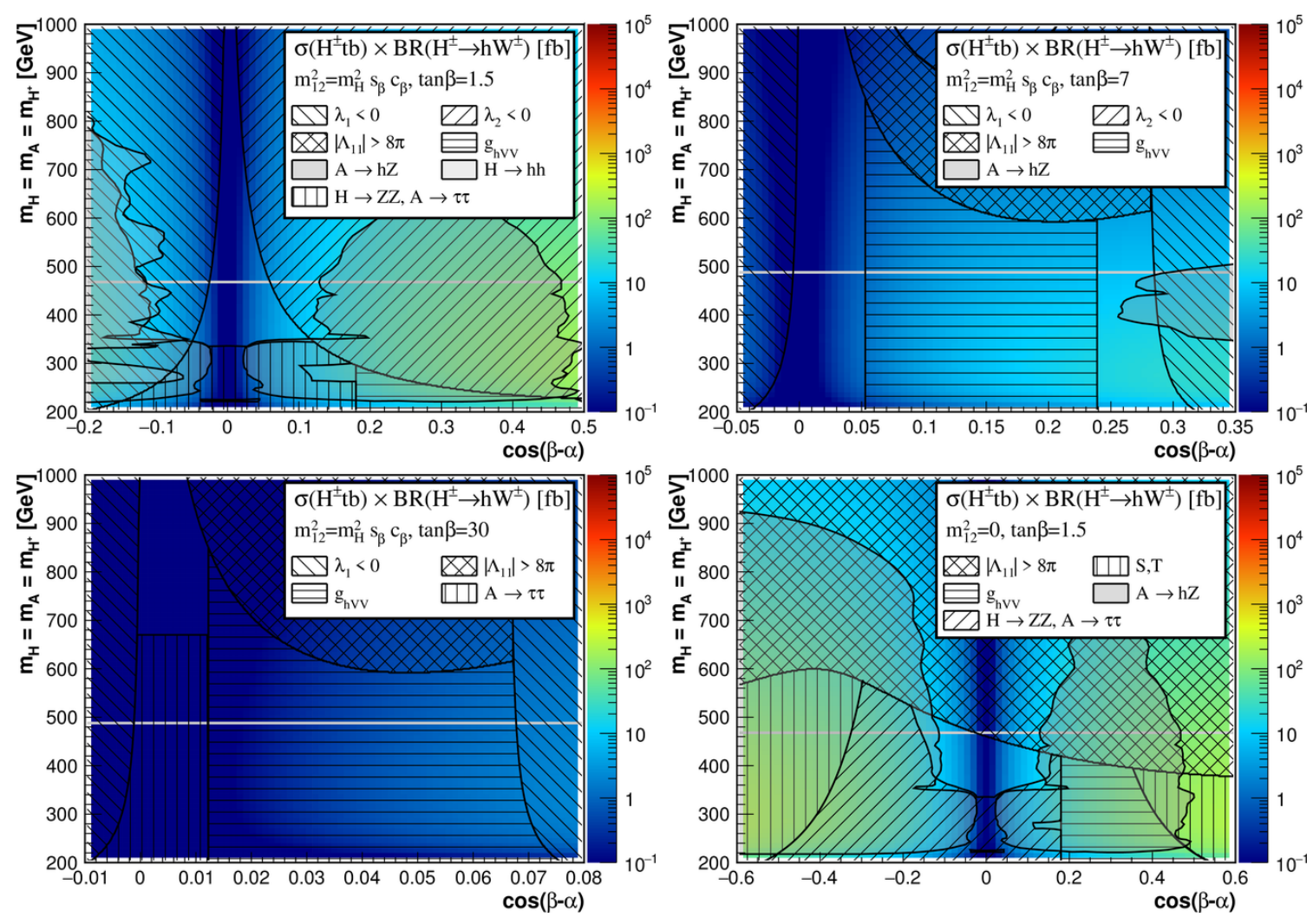

Figure 12. $\sigma \times$ BR for $H^{ \pm} \rightarrow h W^{ \pm}$in BPIII: $c_{\beta-\alpha}$ vs. $m_{A}=m_{H}=m_{H^{ \pm}}$(see caption of figure 11 for further details).

Away from the 2HDM alignment limit, exotic decays into the SM-like $125 \mathrm{GeV}$ Higgs boson $h$, namely $H \rightarrow h h, A \rightarrow h Z$ and $H^{ \pm} \rightarrow h W^{ \pm}$, are potentially important, and there is already an ongoing ATLAS and CMS search programme for $A \rightarrow h Z[55,56]$ and $H \rightarrow h h$ [57-59]. In contrast, close to the alignment limit, as favoured by measurements of Higgs signal strengths, exotic decays among the new 2HDM scalars become particularly relevant. The experimental searches based on those channels, however, have just started with $H / A \rightarrow A Z / H Z[26,27]$. In this work, we carefully examine the exotic Higgs decay channels in the $2 \mathrm{HDM}$, both in the presence of a hierarchy between Higgses and away from alignment when this hierarchy is not present. By taking into account the various theoretical and experimental constraints, we propose 2HDM benchmark plane scenarios for LHC searches at $13 \mathrm{TeV}$ :

- BP IA: $m_{A}>m_{H}=m_{H^{ \pm}}$, with $A \rightarrow H Z, H^{ \pm} W^{\mp}$.

- BP IB: $m_{A}<m_{H}=m_{H^{ \pm}}$, with $H \rightarrow A Z, A A$ and $H^{ \pm} \rightarrow A W^{ \pm}$.

- BP IIA: $m_{H}>m_{A}=m_{H^{ \pm}}$, with $H \rightarrow A Z, H^{ \pm} W^{\mp}, A A, H^{+} H^{-}$.

- BP IIB: $m_{H}<m_{A}=m_{H^{ \pm}}$, with $A \rightarrow H Z$ and $H^{ \pm} \rightarrow H W^{ \pm}$.

- BP III: $m_{A}=m_{H}=m_{H^{ \pm}}$vs. $c_{\beta-\alpha}$, with $A \rightarrow h Z, H^{ \pm} \rightarrow h W^{ \pm}$, and $H \rightarrow h h$. 

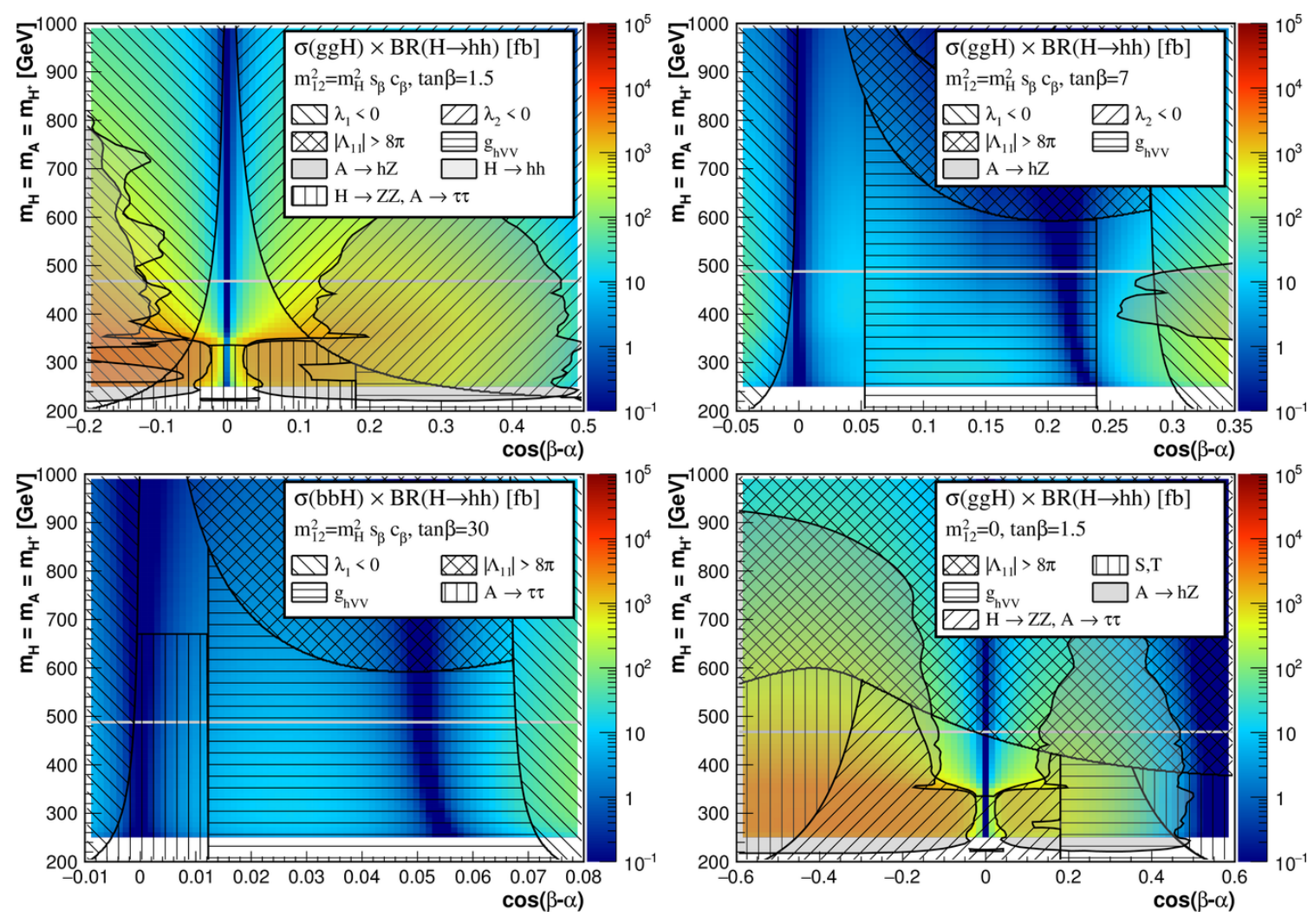

Figure 13. $\sigma \times$ BR for $H \rightarrow h h$ in BPIII: $c_{\beta-\alpha}$ vs. $m_{A}=m_{H}=m_{H^{ \pm}}$(see caption of figure 11 for further details).

In each case, we analyze the allowed regions of parameter space and the $\mathrm{LHC} 13 \mathrm{TeV} \sigma \times \mathrm{BR}$ for the relevant exotic Higgs decay modes in those regions.

To summarize, exotic Higgs decays provide new discovery avenues for heavy Higgses. In turn, the exploration of the proposed benchmarks via these decays could help to understand the structure of the electroweak symmetry breaking sector beyond the SM.

\section{Acknowledgments}

We would like to thank Baradhwaj Coleppa, Tao Han, Tao Liu, Ken Mimasu and Adarsh Pyarelal for helpful discussions. We also thank the Munich Institute for Astro- and Particle Physics (MIAPP) of the DFG cluster of excellence "Origin and Structure of the Universe" for support and hospitality during the initial stages of this work. The work of S.S. and F.K. was supported by US Department of Energy under Grant DE-FG02-04ER-41298. F.K. also acknowledges support from the Fermilab Graduate Student Research Program in Theoretical Physics operated by Fermi Research Alliance, LLC under Contract No. DEAC02-07CH11359 with the United States Department of Energy. J.M.N. is supported by the People Programme (Marie Curie Actions) of the European Union Seventh Framework Programme (FP7/2007-2013) under REA grant agreement PIEF-GA-2013-625809. 

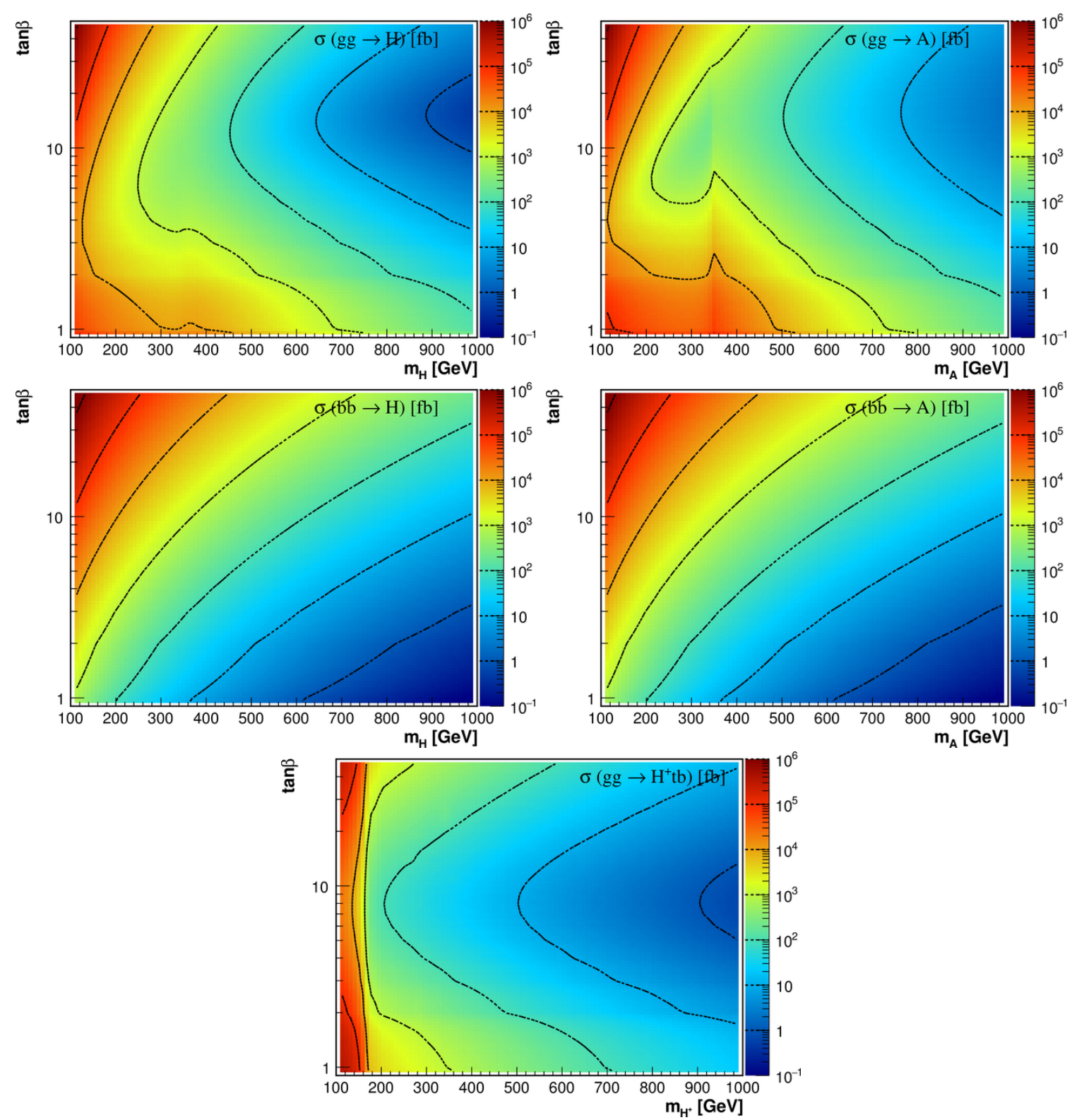

Figure 14. Production cross section for $H, A$ and $H^{+}$at LHC $13 \mathrm{TeV}$. The contour lines indicate the cross section of $1,10,10^{2}, 10^{3}, 10^{4}, 10^{5}$ and $10^{6} \mathrm{fb}$.

\section{A Production cross sections and branching ratios of 2HDM Higgses}

\section{A.1 2HDM production cross sections}

In figure 14, we show the gluon fusion production cross section for $H$ (upper left panel) and $A$ (upper right panel), $b b$-associated production cross section for $H$ (middle left panel) and $A$ (middle right panel), and $t b H^{ \pm}$production cross section (bottom) for the charged scalar (details are given in section 4 ). 

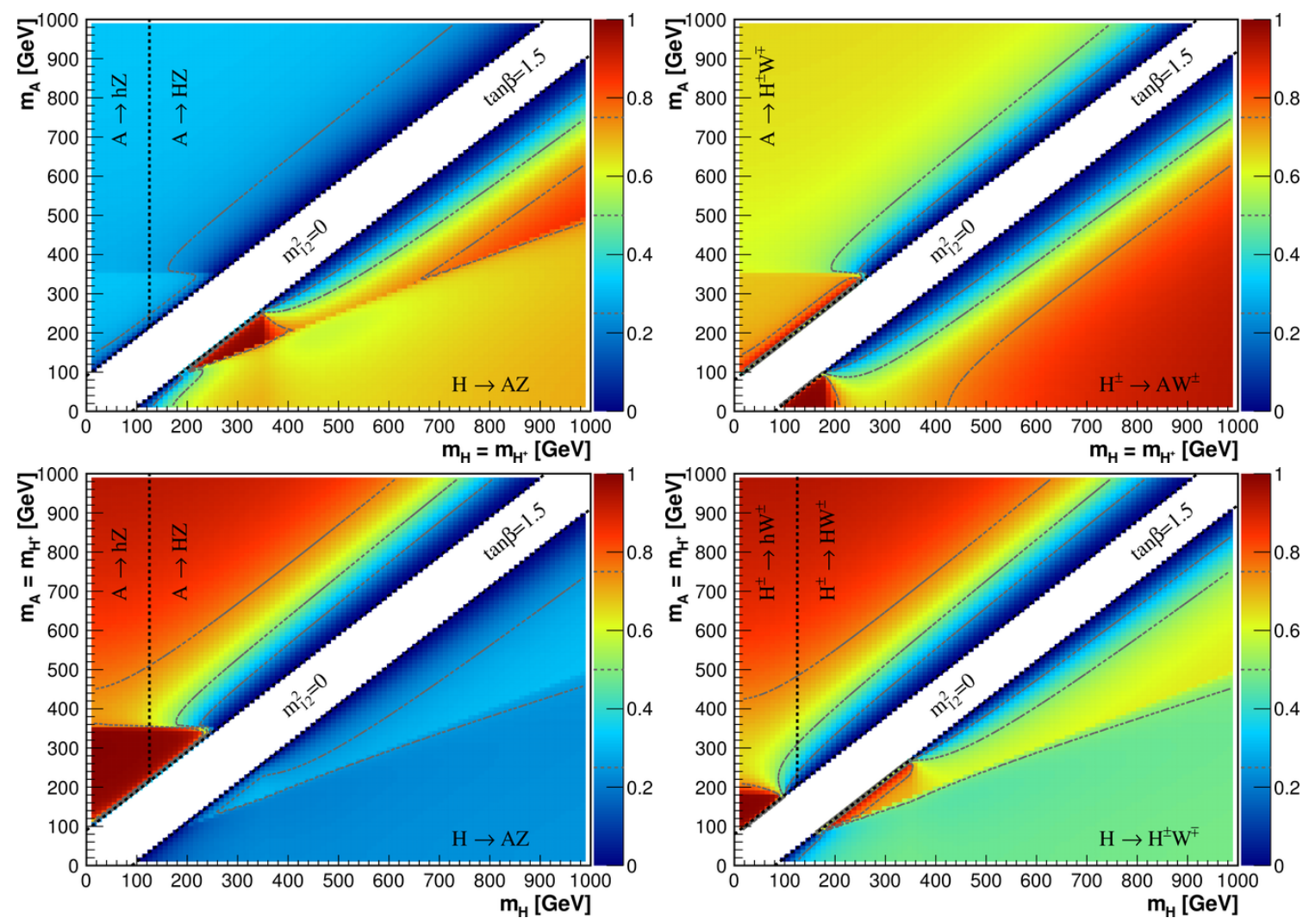

Figure 15. Left: exotic decay BR $H / A \rightarrow A Z / H(h) Z$ for Plane I (top) and Plane II (bottom) for Case $2\left(m_{12}^{2}=0\right)$ with $t_{\beta}=1.5$. For $A \rightarrow H(h) Z$, we consider $A \rightarrow H Z$ for $m_{H}>m_{h}=125 \mathrm{GeV}$ and $A \rightarrow h Z$ for $m_{h}<m_{H}=125 \mathrm{GeV}$ (so that the BR into the non-SM like Higgs boson is shown in each case). Right: exotic decay BR $A / H^{ \pm} \rightarrow H^{ \pm} W^{\mp} / A W^{ \pm}$for Plane I (top) and $H / H^{ \pm} \rightarrow H^{ \pm} W^{\mp} / H W^{ \pm}$for Plane II (bottom), for Case $2\left(m_{12}^{2}=0\right)$ with $t_{\beta}=1.5$.

\section{A.2 2HDM branching ratios for exotic Higgs decays}

For illustration, we show in figure 15 the branching ratios of $H_{a} \rightarrow H_{b} V$ (with $H_{a, b}=$ $H, A, H^{ \pm}$and $\left.V=W^{ \pm}, Z\right)$ for Plane I (top) and Plane II (bottom) for Case $2\left(m_{12}^{2}=0\right)$ with $t_{\beta}=1.5$ (being the scenario allowed for the four benchmarks BP IA, BP IB, BP IIA and BP IIB). The decay branching ratios for $H / A \rightarrow A Z / H(h) Z$ are shown on the left panels of figure 15, while those for $A / H^{ \pm} \rightarrow H^{ \pm} W^{\mp} / A W^{ \pm}$(Plane I) and $H / H^{ \pm} \rightarrow$ $H^{ \pm} W^{\mp} / H W^{ \pm}$(Plane II) are shown on the right panels. 
Open Access. This article is distributed under the terms of the Creative Commons Attribution License (CC-BY 4.0), which permits any use, distribution and reproduction in any medium, provided the original author(s) and source are credited.

\section{References}

[1] ATLAS collaboration, Measurements of the Higgs boson production and decay rates and coupling strengths using pp collision data at $\sqrt{s}=7$ and $8 \mathrm{TeV}$ in the ATLAS experiment, Eur. Phys. J. C 76 (2016) 6 [arXiv:1507.04548] [InSPIRE].

[2] CMS collaboration, Precise determination of the mass of the Higgs boson and tests of compatibility of its couplings with the standard model predictions using proton collisions at 7 and $8 \mathrm{TeV}$, Eur. Phys. J. C 75 (2015) 212 [arXiv:1412.8662] [inSPIRE].

[3] A. Djouadi, The anatomy of electro-weak symmetry breaking. II. The Higgs bosons in the minimal supersymmetric model, Phys. Rept. 459 (2008) 1 [hep-ph/0503173] [INSPIRE].

[4] J. Mrazek, A. Pomarol, R. Rattazzi, M. Redi, J. Serra and A. Wulzer, The Other Natural Two Higgs Doublet Model, Nucl. Phys. B 853 (2011) 1 [arXiv:1105.5403] [INSPIRE].

[5] E. Bertuzzo, T.S. Ray, H. de Sandes and C.A. Savoy, On Composite Two Higgs Doublet Models, JHEP 05 (2013) 153 [arXiv:1206.2623] [INSPIRE].

[6] J.M. Cline, K. Kainulainen and A.P. Vischer, Dynamics of two Higgs doublet CP-violation and baryogenesis at the electroweak phase transition, Phys. Rev. D 54 (1996) 2451 [hep-ph/9506284] [INSPIRE].

[7] J.M. Cline and P.-A. Lemieux, Electroweak phase transition in two Higgs doublet models, Phys. Rev. D 55 (1997) 3873 [hep-ph/9609240] [INSPIRE].

[8] L. Fromme, S.J. Huber and M. Seniuch, Baryogenesis in the two-Higgs doublet model, JHEP 11 (2006) 038 [hep-ph/0605242] [INSPIRE].

[9] G.C. Dorsch, S.J. Huber and J.M. No, A strong electroweak phase transition in the 2HDM after LHC8, JHEP 10 (2013) 029 [arXiv:1305.6610] [INSPIRE].

[10] A. Celis, V. Ilisie and A. Pich, LHC constraints on two-Higgs doublet models, JHEP 07 (2013) 053 [arXiv: 1302.4022] [INSPIRE].

[11] B. Grinstein and P. Uttayarat, Carving Out Parameter Space in Type-II Two Higgs Doublets Model, JHEP 06 (2013) 094 [Erratum ibid. 09 (2013) 110] [arXiv: 1304.0028] [INSPIRE].

[12] B. Coleppa, F. Kling and S. Su, Constraining Type II 2HDM in Light of LHC Higgs Searches, JHEP 01 (2014) 161 [arXiv:1305.0002] [INSPIRE].

[13] C.-Y. Chen, S. Dawson and M. Sher, Heavy Higgs Searches and Constraints on Two Higgs Doublet Models, Phys. Rev. D 88 (2013) 015018 [Erratum ibid. D 88 (2013) 039901] [arXiv: 1305.1624] [INSPIRE].

[14] O. Eberhardt, U. Nierste and M. Wiebusch, Status of the two-Higgs-doublet model of type-II, JHEP 07 (2013) 118 [arXiv: 1305.1649] [INSPIRE].

[15] B. Dumont, J.F. Gunion, Y. Jiang and S. Kraml, Constraints on and future prospects for Two-Higgs-Doublet Models in light of the LHC Higgs signal, Phys. Rev. D 90 (2014) 035021 [arXiv: 1405.3584] [INSPIRE].

[16] J. Bernon, B. Dumont and S. Kraml, Status of Higgs couplings after run 1 of the LHC, Phys. Rev. D 90 (2014) 071301 [arXiv: 1409.1588] [INSPIRE]. 
[17] N. Craig, F. D'Eramo, P. Draper, S. Thomas and H. Zhang, The Hunt for the Rest of the Higgs Bosons, JHEP 06 (2015) 137 [arXiv: 1504.04630] [INSPIRE].

[18] J. Bernon, J.F. Gunion, H.E. Haber, Y. Jiang and S. Kraml, Scrutinizing the alignment limit in two-Higgs-doublet models: $m_{h}=125$ GeV, Phys. Rev. D 92 (2015) 075004 [arXiv: 1507.00933] [INSPIRE].

[19] G.C. Dorsch, S.J. Huber, K. Mimasu and J.M. No, Hierarchical versus degenerate 2HDM: The LHC run 1 legacy at the onset of run 2, Phys. Rev. D 93 (2016) 115033 [arXiv: 1601.04545] [INSPIRE].

[20] B. Coleppa, F. Kling and S. Su, Exotic Higgs Decay via AZ/HZ Channel: a Snowmass Whitepaper, arXiv:1308.6201 [inSPIRE].

[21] B. Coleppa, F. Kling and S. Su, Exotic Decays Of A Heavy Neutral Higgs Through HZ/AZ Channel, JHEP 09 (2014) 161 [arXiv:1404.1922] [INSPIRE].

[22] G.C. Dorsch, S.J. Huber, K. Mimasu and J.M. No, Echoes of the Electroweak Phase Transition: Discovering a second Higgs doublet through $A_{0} \rightarrow Z H_{0}$, Phys. Rev. Lett. 113 (2014) 211802 [arXiv:1405.5537] [INSPIRE].

[23] B. Coleppa, F. Kling and S. Su, Charged Higgs search via $A W^{ \pm} / H W^{ \pm}$channel, JHEP 12 (2014) 148 [arXiv: 1408.4119] [InSPIRE].

[24] T. Li and S. Su, Exotic Higgs Decay via Charged Higgs, JHEP 11 (2015) 068 [arXiv: 1504.04381] [INSPIRE].

[25] F. Kling, A. Pyarelal and S. Su, Light Charged Higgs Bosons to AW/HW via Top Decay, JHEP 11 (2015) 051 [arXiv: 1504.06624] [INSPIRE].

[26] CMS collaboration, Search for $H / A$ decaying into $Z+A / H$, with $Z$ to $l l$ and $A / H$ to fermion pair, CMS-PAS-HIG-15-001.

[27] CMS collaboration, Search for neutral resonances decaying into a $Z$ boson and a pair of $b$ jets or tau leptons, Phys. Lett. B 759 (2016) 369 [arXiv:1603.02991] [INSPIRE].

[28] J.F. Gunion, H.E. Haber, G.L. Kane and S. Dawson, The Higgs Hunter's Guide, Front. Phys. 80 (2000) 1 [INSPIRE].

[29] J. Bernon, J.F. Gunion, H.E. Haber, Y. Jiang and S. Kraml, Scrutinizing the alignment limit in two-Higgs-doublet models. II. $m_{H}=125 \mathrm{GeV}$, Phys. Rev. D 93 (2016) 035027 [arXiv: 1511.03682] [INSPIRE].

[30] J.F. Gunion and H.E. Haber, The CP conserving two Higgs doublet model: The approach to the decoupling limit, Phys. Rev. D 67 (2003) 075019 [hep-ph/0207010] [INSPIRE].

[31] LEP, DELPHI, OPAL, ALEPH, L3 collaborations, G. Abbiendi et al., Search for Charged Higgs bosons: Combined Results Using LEP Data, Eur. Phys. J. C 73 (2013) 2463 [arXiv: 1301.6065] [INSPIRE].

[32] J. Bernon, J.F. Gunion, Y. Jiang and S. Kraml, Light Higgs bosons in Two-Higgs-Doublet Models, Phys. Rev. D 91 (2015) 075019 [arXiv:1412.3385] [INSPIRE].

[33] S.L. Glashow and S. Weinberg, Natural Conservation Laws for Neutral Currents, Phys. Rev. D 15 (1977) 1958 [INSPIRE].

[34] G.C. Branco, P.M. Ferreira, L. Lavoura, M.N. Rebelo, M. Sher and J.P. Silva, Theory and phenomenology of two-Higgs-doublet models, Phys. Rept. 516 (2012) 1 [arXiv:1106.0034] [INSPIRE]. 
[35] ATLAS collaboration, Constraints on new phenomena via Higgs boson couplings and invisible decays with the ATLAS detector, JHEP 11 (2015) 206 [arXiv:1509.00672] [INSPIRE].

[36] P.M. Ferreira, J.F. Gunion, H.E. Haber and R. Santos, Probing wrong-sign Yukawa couplings at the LHC and a future linear collider, Phys. Rev. D 89 (2014) 115003 [arXiv:1403.4736] [INSPIRE].

[37] I.F. Ginzburg and I.P. Ivanov, Tree-level unitarity constraints in the most general 2HDM, Phys. Rev. D 72 (2005) 115010 [hep-ph/0508020] [InSPIRE].

[38] B. Grinstein, C.W. Murphy and P. Uttayarat, One-loop corrections to the perturbative unitarity bounds in the CP-conserving two-Higgs doublet model with a softly broken $\mathbb{Z}_{2}$ symmetry, JHEP 06 (2016) 070 [arXiv:1512.04567] [INSPIRE].

[39] GFitTer Group collaboration, M. Baak et al., The global electroweak fit at NNLO and prospects for the LHC and ILC, Eur. Phys. J. C 74 (2014) 3046 [arXiv:1407.3792] [INSPIRE].

[40] M. Gorbahn, J.M. No and V. Sanz, Benchmarks for Higgs Effective Theory: Extended Higgs Sectors, JHEP 10 (2015) 036 [arXiv: 1502.07352] [InSPIRE].

[41] Heavy Flavor Averaging Group (HFAG) collaboration, Y. Amhis et al., Averages of b-hadron, c-hadron and $\tau$-lepton properties as of summer 2014, arXiv:1412.7515 [INSPIRE].

[42] F. Mahmoudi, SuperIso v2.3: A program for calculating flavor physics observables in Supersymmetry, Comput. Phys. Commun. 180 (2009) 1579 [arXiv:0808.3144] [INSPIRE].

[43] F. Mahmoudi, SuperIso v3.0, flavor physics observables calculations: Extension to NMSSM, Comput. Phys. Commun. 180 (2009) 1718 [INSPIRE].

[44] BaBAr collaboration, J.P. Lees et al., Evidence for an excess of $\bar{B} \rightarrow D^{(*)} \tau^{-} \bar{\nu}_{\tau}$ decays, Phys. Rev. Lett. 109 (2012) 101802 [arXiv:1205.5442] [INSPIRE].

[45] BeLle collaboration, M. Huschle et al., Measurement of the branching ratio of $\bar{B} \rightarrow D^{(*)} \tau^{-} \bar{\nu}_{\tau}$ relative to $\bar{B} \rightarrow D^{(*)} \ell^{-} \bar{\nu}_{\ell}$ decays with hadronic tagging at Belle, Phys. Rev. $\mathbf{D}$ 92 (2015) 072014 [arXiv: 1507.03233] [INSPIRE].

[46] Belle collaboration, A. Abdesselam et al., Measurement of the branching ratio of $\bar{B}^{0} \rightarrow D^{*+} \tau^{-} \bar{\nu}_{\tau}$ relative to $\bar{B}^{0} \rightarrow D^{*+} \ell^{-} \bar{\nu}_{\ell}$ decays with a semileptonic tagging method, arXiv:1603.06711 [INSPIRE].

[47] M. Misiak et al., Updated NNLO QCD predictions for the weak radiative B-meson decays, Phys. Rev. Lett. 114 (2015) 221801 [arXiv:1503.01789] [INSPIRE].

[48] T. Han, T. Li, S. Su and L.-T. Wang, Non-Decoupling MSSM Higgs Sector and Light Superpartners, JHEP 11 (2013) 053 [arXiv:1306.3229] [InSPIRE].

[49] DELPHI, OPAL, ALEPH, L3, LEP Working Group for Higgs Boson Searches, collaborations, S. Schael et al., Search for neutral MSSM Higgs bosons at LEP, Eur. Phys. J. C 47 (2006) 547 [hep-ex/0602042] [INSPIRE].

[50] ATLAS collaboration, Search for neutral Higgs bosons of the minimal supersymmetric standard model in pp collisions at $\sqrt{s}=8 \mathrm{TeV}$ with the ATLAS detector, JHEP 11 (2014) 056 [arXiv: 1409.6064] [INSPIRE].

[51] CMS collaboration, Search for neutral MSSM Higgs bosons decaying to a pair of tau leptons in pp collisions, JHEP 10 (2014) 160 [arXiv:1408.3316] [INSPIRE]. 
[52] ATLAS collaboration, Measurements of the properties of the Higgs-like boson in the four lepton decay channel with the ATLAS detector using $25 \mathrm{fb}-1$ of proton-proton collision data, ATLAS-CONF-2013-013 (2013).

[53] ATLAS collaboration, Observation and measurement of Higgs boson decays to $W W^{*}$ with the ATLAS detector, Phys. Rev. D 92 (2015) 012006 [arXiv:1412.2641] [INSPIRE].

[54] CMS collaboration, Search for a Higgs Boson in the Mass Range from 145 to $1000 \mathrm{GeV}$ Decaying to a Pair of $W$ or $Z$ Bosons, JHEP 10 (2015) 144 [arXiv: 1504.00936] [InSPIRE].

[55] ATLAS collaboration, Search for a CP-odd Higgs boson decaying to Zh in pp collisions at $\sqrt{s}=8 \mathrm{TeV}$ with the ATLAS detector, Phys. Lett. B 744 (2015) 163 [arXiv:1502.04478] [INSPIRE].

[56] CMS collaboration, Search for a pseudoscalar boson decaying into a $Z$ boson and the $125 \mathrm{GeV}$ Higgs boson in $\ell^{+} \ell^{-} b \bar{b}$ final states, Phys. Lett. B 748 (2015) 221 [arXiv: 1504.04710] [INSPIRE].

[57] ATLAS collaboration, Search For Higgs Boson Pair Production in the $\gamma \gamma b \bar{b}$ Final State using pp Collision Data at $\sqrt{s}=8 \mathrm{TeV}$ from the ATLAS Detector, Phys. Rev. Lett. 114 (2015) 081802 [arXiv: 1406.5053] [INSPIRE].

[58] CMS collaboration, Search for resonant HH production in 2gamma+2b channel, CMS-PAS-HIG-13-032.

[59] CMS collaboration, Search for resonant pair production of Higgs bosons decaying to two bottom quark-antiquark pairs in proton-proton collisions at 8 TeV, Phys. Lett. B 749 (2015) 560 [arXiv: 1503.04114] [inSPIRE].

[60] ATLAS collaboration, Search for charged Higgs bosons decaying via $H^{ \pm} \rightarrow \tau^{ \pm} \nu$ in fully hadronic final states using pp collision data at $\sqrt{s}=8 \mathrm{TeV}$ with the ATLAS detector, JHEP 03 (2015) 088 [arXiv: 1412.6663] [INSPIRE].

[61] CMS collaboration, Search for a charged Higgs boson in pp collisions at $\sqrt{s}=8 \mathrm{TeV}$, JHEP 11 (2015) 018 [arXiv: 1508.07774] [INSPIRE].

[62] D. Das and I. Saha, Search for a stable alignment limit in two-Higgs-doublet models, Phys. Rev. D 91 (2015) 095024 [arXiv: 1503.02135] [INSPIRE].

[63] D. Das, New limits on tan $\beta$ for 2HDMs with $Z_{2}$ symmetry, Int. J. Mod. Phys. A 30 (2015) 1550158 [arXiv: 1501.02610 ] [INSPIRE].

[64] R.V. Harlander, S. Liebler and H. Mantler, SusHi: A program for the calculation of Higgs production in gluon fusion and bottom-quark annihilation in the Standard Model and the MSSM, Comput. Phys. Commun. 184 (2013) 1605 [arXiv:1212.3249] [INSPIRE].

[65] M. Flechl, R. Klees, M. Krämer, M. Spira and M. Ubiali, Improved cross-section predictions for heavy charged Higgs boson production at the LHC, Phys. Rev. D 91 (2015) 075015 [arXiv: 1409.5615] [INSPIRE].

[66] LHC Higgs Cross Section Working Group collaboration, Handbook of LHC Higgs Cross sections: 3. Higgs Properties, arXiv:1307.1347 [INSPIRE].

[67] S. Dittmaier, M. Krämer, M. Spira and M. Walser, Charged-Higgs-boson production at the LHC: NLO supersymmetric QCD corrections, Phys. Rev. D 83 (2011) 055005 [arXiv:0906.2648] [INSPIRE]. 
[68] E.L. Berger, T. Han, J. Jiang and T. Plehn, Associated production of a top quark and a charged Higgs boson, Phys. Rev. D 71 (2005) 115012 [hep-ph/0312286] [INSPIRE].

[69] M. Czakon and A. Mitov, Top++: A Program for the Calculation of the Top-Pair Cross-Section at Hadron Colliders, Comput. Phys. Commun. 185 (2014) 2930 [arXiv:1112.5675] [INSPIRE].

[70] D. Eriksson, J. Rathsman and O. Stål, 2HDMC: Two-Higgs-Doublet Model Calculator Physics and Manual, Comput. Phys. Commun. 181 (2010) 189 [arXiv:0902.0851] [InSPIRE].

[71] H.E. Haber and O. Stål, New LHC benchmarks for the $\mathcal{C} \mathcal{P}$-conserving two-Higgs-doublet model, Eur. Phys. J. C 75 (2015) 491 [arXiv:1507.04281] [INSPIRE].

[72] C. Gao, M.A. Luty, M. Mulhearn, N.A. Neill and Z. Wang, Searching for Additional Higgs Bosons via Higgs Cascades, arXiv:1604.03108 [INSPIRE].

[73] M. Bauer, M. Carena and K. Gemmler, Flavor from the Electroweak Scale, JHEP 11 (2015) 016 [arXiv: 1506.01719] [INSPIRE]. 